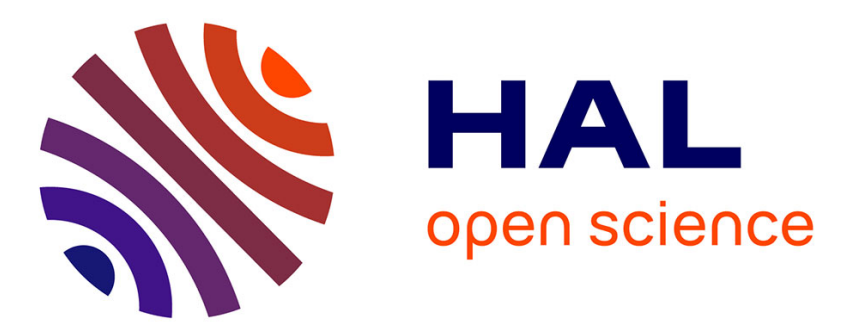

\title{
Pyrometer model based on sensor physical structure and thermal operation
}

\author{
Eduardo Sebastián, Carlos Armiens, Javier Gómez-Elvira
}

\section{To cite this version:}

Eduardo Sebastián, Carlos Armiens, Javier Gómez-Elvira. Pyrometer model based on sensor physical structure and thermal operation. Applied Thermal Engineering, 2010, 30 (16), pp.2403. 10.1016/j.applthermaleng.2010.06.010 . hal-00675400

\section{HAL Id: hal-00675400 https://hal.science/hal-00675400}

Submitted on 1 Mar 2012

HAL is a multi-disciplinary open access archive for the deposit and dissemination of scientific research documents, whether they are published or not. The documents may come from teaching and research institutions in France or abroad, or from public or private research centers.
L'archive ouverte pluridisciplinaire HAL, est destinée au dépôt et à la diffusion de documents scientifiques de niveau recherche, publiés ou non, émanant des établissements d'enseignement et de recherche français ou étrangers, des laboratoires publics ou privés. 


\section{Accepted Manuscript}

Title: Pyrometer model based on sensor physical structure and thermal operation

Authors: Eduardo Sebastián, Carlos Armiens, Javier Gómez-Elvira

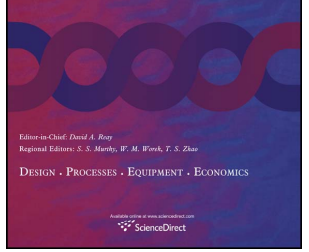

PII:

$$
\text { S1359-4311(10)00257-7 }
$$

DOI: $\quad$ 10.1016/j.applthermaleng.2010.06.010

Reference: $\quad$ ATE 3141

To appear in: Applied Thermal Engineering

Received Date: 24 November 2009

Revised Date: 15 March 2010

Accepted Date: 14 June 2010

Please cite this article as: E. Sebastián, C. Armiens, J. Gómez-Elvira. Pyrometer model based on sensor physical structure and thermal operation, Applied Thermal Engineering (2010), doi: 10.1016/ j.applthermaleng.2010.06.010

This is a PDF file of an unedited manuscript that has been accepted for publication. As a service to our customers we are providing this early version of the manuscript. The manuscript will undergo copyediting, typesetting, and review of the resulting proof before it is published in its final form. Please note that during the production process errors may be discovered which could affect the content, and all legal disclaimers that apply to the journal pertain. 


\title{
Pyrometer model based on sensor physical structure and thermal
}

\section{operation}

\author{
Eduardo Sebastián, Carlos Armiens, Javier Gómez-Elvira
}

Laboratorio de Robótica y Exploración Planetaria, Centro de Astrobiología (CSIC-INTA) ${ }^{1}$

\begin{abstract}
This paper proposes a new simplified thermal model for pyrometers, which takes into account both their internal and external physical structure and operation. The model is experimentally tested on the REMS GTS, an instrument for measuring ground temperature, which is part of the payload of the NASA MSL mission to Mars. The proposed model is based on an energy balance equation that represents the heat fluxes exchanged between sensor elements through radiation, conduction and convection. Despite being mathematically more complex than the more commonly used model, the proposed model makes it possible to design a methodology to compensate the effects of sensor spatial thermal gradients. The paper includes a practical methodology for identifying model constants, which is part of the GTS instrument calibration plan and uses a differential approach to avoid setup errors. Experimental results of the model identification methodology and a target temperature measurement performance after identification has been made are reported. Results demonstrate the good behaviour of the model, with errors below $0.15^{\circ} \mathrm{C}$ in target temperature estimates.
\end{abstract}

Keywords: IR temperature sensor; Sensor thermal model; Sensor calibration; Spacecraft instrumentation

\section{Introduction}

In the study and design of IR sensors, and more specifically of thermopiles, physical models of their internal structure are used [2][3][5]. Amongst other parameters, these models take into account: the thermal conductivities of the construction materials, convection and radiation heat

\footnotetext{
${ }^{1}$ Ctra. Ajalvir Km.4, Torrejón de Ardoz, Spain, Telf.: +34 915206405 Fax: +34 915201074

Email: sebastianme@inta.es
} 
fluxes inside the thermopile and the geometrical disposition, mass and volume of the sensor elements. The objective is to calculate and design the sensibility and time response of the sensor based on the value of these parameters.

This study is unlike the work usually carried out with IR sensors as pyrometer, which uses sensor models that do not take into account the internal structure of the thermopile [4][5]. The usual way of dealing with these sensors is through an equation that associates the electrical output variable of the transducer with the difference in radiated energy between the transducer and the target using a gain, the value of which depends on the temperature of the transducer [2]. The main advantage of this simple model is that it is easy to implement in practical applications, does not require high computational capabilities, and in some cases a simple analogue amplifier is enough to obtain a representation of the target temperature [6]. Other authors [1] propose a direct polynomial interpolation between the transducer output voltage and temperature with the target temperature, obtaining high accuracy. The main drawback with all these models is that they do not include a representation of the physical structure and operation of the sensor, as there is no direct relation between any physical property and the model parameters.

Taking into account the structure of the internal IR sensor in the model sensor may help us to understand its behaviour after its change or degradation, or the appearance of temperature gradients in its structure, being the capability of establishing calibration or compensation algorithms for those parameters that are directly related to sensor physical behaviour. For instance, in [8] the author proposes an internal thermal equilibrium equation, for different kinds of unchopped thermal detectors, to model its sensibility to changes in instrument temperature. The authors of [12] go beyond that in an attempt to establish a methodology, also based on a simplified internal model of a thermopile, which avoids errors that stem from sensor temperature instabilities or temporal gradients. In [13] the author proposes an IR sensor concept, based on its internal operation, which accounts for the sensor's behaviour under the existence of spatial temperature gradients on its can. Finally, in [7] the authors discuss a thermal model for a thermo electric flux meter, consisting of a thermoelectric device and based on its physical construction and parameters. 
The main contribution of this article is the proposal of a new simplified analytical thermal model for pyrometers based on the sensor's physical structure and operation. The proposed model accounts for the heat fluxes exchanged between the sensor's transducer and the rest of its elements through radiation, conduction and convection. It also includes an estimator to compensate for the appearance of the thermopile's spatial thermal gradients, but contrary to the generic solution given in [13], this estimator makes use of the temperature evolution of an external temperature sensor. With the model a differential methodology to calibrate its constants can be established. The experimental data corroborate the ability of the proposed model to represent sensor behaviour, identifying model constants and showing its ability to measure the temperature of a target surface.

\subsection{Brief description of REMS GTS}

This study was originally motivated by the application of a pyrometer in space, the Ground Temperature Sensor (GTS) of the Rover Environmental Monitoring Station (REMS) [14], which is part of the NASA Mars Science Laboratory (MSL) mission to Mars [9]. This pyrometer is used to measure kinematic ground temperature, integrating the radiation of the Martian surface in three different infrared wavelength channels. The GTS has a total size of 40x28x19mm and a weight bellow 20g, see Fig.1. It is formed by three TS-100 thermopiles supplied by the company IPHT Jena [10], encapsulated inside a TO-5 and facing the ground with no optical system apart from the thermopile filter. The thermopiles have an internal Resistance Temperature Detector (RTD) to measure the temperature of the base of their can $T_{c b}$, which acts as the cold junction reference temperature of the thermocouples, and a non corrosive, insulating and transparent filling atmosphere. The thermopiles are situated inside a metal piece used as a thermal mass to minimize the temporal and spatial drift in their temperature [12].

In remote or hostile applications, recalibration and optic cleaning systems are key features of any pyrometer. In commercial applications designers usually resort to cleaning systems based on artificial air purges to avoid optic deterioration. Due to space resource restrictions the GTS includes an in-flight calibration system with no moving parts, which accounts for the degradation of the sensor due to dust deposits on the thermopile's filter. The system is 
implemented by a high emissivity and low mass calibration plate; see Fig. 1, which can be heated to the desired temperature, using for that only $0.5 \mathrm{~W}$ during 5 minutes. It is placed in front of the thermopiles' hosting piece, so that each thermopile faces the ground through a hole in this plate. In this way an annulus of the filter that represents part of the thermopiles' FOV is obstructed by the calibration system, limiting the solid angle measurement. The temperature of this plate, $T_{p}$, is measured using a specific RTD glued to its surface. As far as the authors are aware, this is the first time a recalibration system like the one described here and without moving parts has been used in this way on a pyrometer.

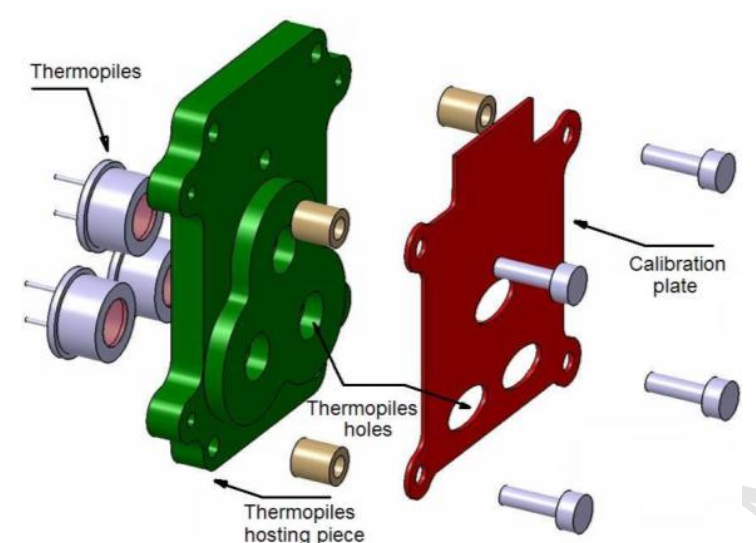

(a)

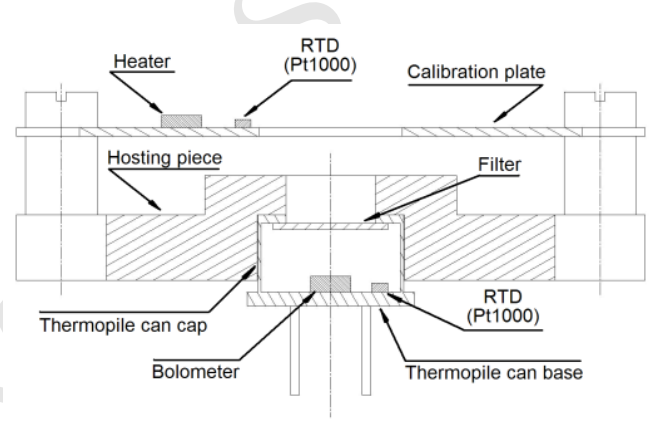

(b)

Fig.1 (a) The 3D mechanical layout of REMS GTS, (b)The 2D mechanical layout of REMS GTS and a thermopile.

It is important to note the existence of potential applications on our planet, in relation to the specific characteristics of the GTS autonomous recalibration system. Some examples would be air condition systems or temperature monitoring of train and car wheels and brakes, in general commercial applications with intensive use of contactless temperature sensors where recalibration of individual sensors would imply a high cost. Also, environmental monitoring systems deployed in extreme and remote places with long working times, where access for recalibrating is complicated and expensive.

\section{GTS thermal model}

The proposed thermal model is based on an energy balance equation (1) that accounts for the heat in the thermopile bolometer coming from all the bodies around it, see Fig.2a, ignoring the 
heat exchanged between bodies other than the bolometer, but taking into account in an implicit

way a certain influence of the internal reflections inside the thermopile's can.

$$
P_{R, g-s}+P_{R, p-s}+P_{R, f-s}+P_{R, c c-s}+P_{R, c b-s}+P_{C, c b-s}=C \frac{d T_{s}}{d t}
$$

$P_{R}$ and $P_{C}$ are terms that represent the heat exchanged between the bodies of the subscripts,

through radiation, conduction and convection respectively. As the bolometer is designed to be well insulated from the can and to have low thermal mass, the thermal equilibrium condition of the equation (1) is reached after a setting time of a few milliseconds, which means $C \frac{d T_{s}}{d t}=0$.

\section{Nomenclature}

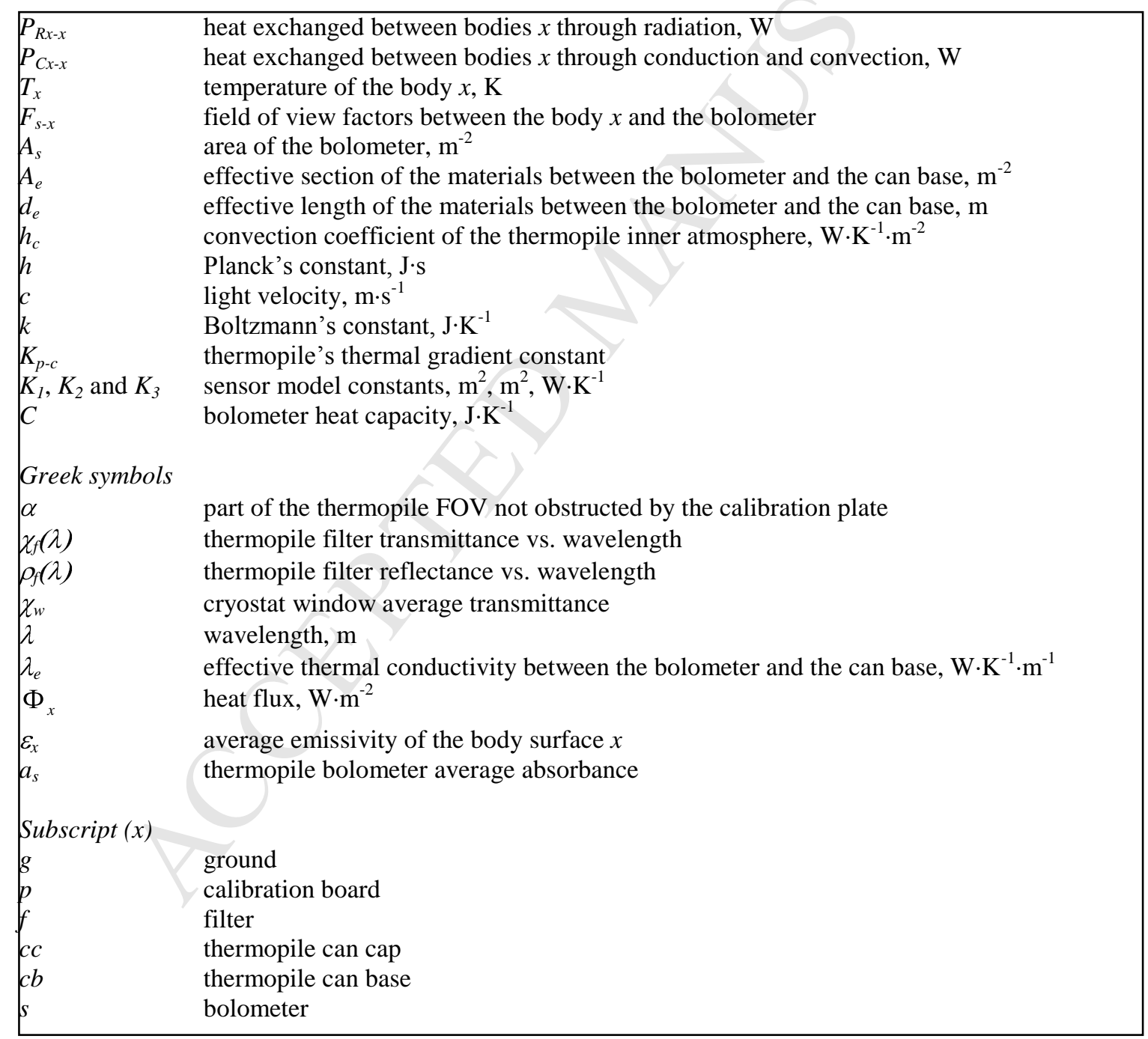

Based on simplified one dimensional heat transfer models using the standard convection, conduction and radiation expression [11], in which it is assumed that the temperature of the atmosphere inside the thermopile is equal to that of the can base, $T_{c b}$, and that thermal 
equilibrium is reached, equation (1) can be rewritten in (2). Heat flux terms $\Phi_{x}$ are calculated based on Planck's law.

$A_{s} \cdot F_{s-g} \cdot \Phi_{g}+A_{s} \cdot F_{s-p} \cdot \Phi_{p}+A_{s} \cdot F_{s-f} \cdot \Phi_{f}+A_{s} \cdot F_{s-c c} \cdot \Phi_{c c}+A_{s} \cdot F_{s-c b} \cdot \Phi_{c b}-A_{s} \cdot\left(F_{s-f}+F_{s-c b}+F_{s-c c}\right) \cdot \Phi_{s}+\left(\lambda_{e} \cdot \frac{A_{e}}{d_{e}}+2 \cdot h_{c} \cdot A_{s}\right) \cdot\left(T_{c b}-T_{s}\right)=0$

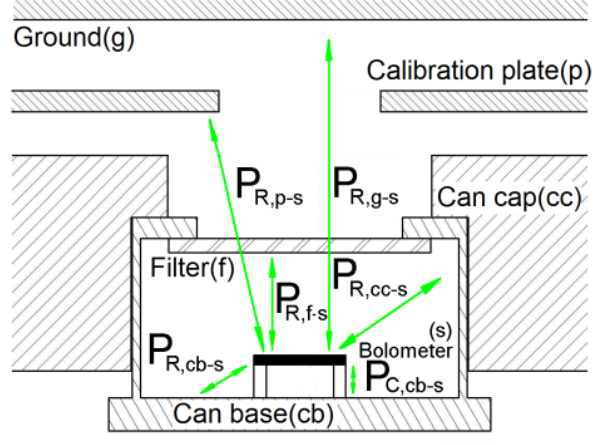

(a)

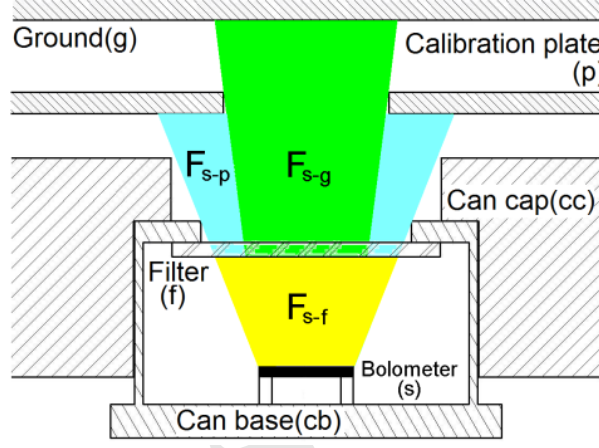

(b)

Fig.2 (a) Thermal diagram of sensor exchanged heat, (b) Diagram of sensor field of view.

Two reasonable simplifications can be assumed in (2). First, that the bolometer's FOV of the filter, which is limited by the shape of the thermopile can, is equal to the sum of the ground and calibration plate FOV $F_{s-f}=F_{s-g}+F_{s-p}$, see Fig. $2 \mathrm{~b}$, and second, the bolometer's FOV of the thermopile's can base and can cap are quite similar $F_{s-c b} \approx F_{s-c c}$ since they represent almost the same approximate solid angle, $2 \pi$ steradian $^{2}$.

Areas, FOV factors, conductivities, convection coefficient and effective length in (2) are initially unknown, and depend on specific parameters of the thermopile materials and their relative position inside the GTS. In order to simplify the process of identifying these terms they have been grouped together, obtaining as a result equation (3)

$$
\alpha \cdot K_{1} \cdot \Phi_{g}+(1-\alpha) \cdot K_{1} \cdot \Phi_{p}+K_{1} \cdot \Phi_{f}+\frac{K_{2}}{2} \cdot \Phi_{c c}+\frac{K_{2}}{2} \cdot \Phi_{c b}-\left(K_{1}+K_{2}\right) \cdot \Phi_{s}+K_{3} \cdot\left(T_{c b}-T_{s}\right)=0
$$

where $K_{l}=A_{s} \cdot\left(F_{s-g}+F_{s-p}\right), K_{2}=A_{s} \cdot\left(F_{s-c c}+F_{s-c b}\right)=, K_{3}=\lambda_{e} \cdot \frac{A_{e}}{d_{e}}+h_{c} \cdot A_{s} \cdot 2$, and $\alpha=\frac{F_{s-g}}{F_{s-g}+F_{s-p}}$.

Equations (4) show the way in which the heat flux terms are calculated, integrating Planck's law. The expressions consider that an incoming radiation becomes a heat gain through the

\footnotetext{
${ }^{2}$ This assumption takes into account the small size of the filter, $2 \mathrm{~mm}$, versus the $8 \mathrm{~mm}$ of thermopile diameter $F_{s-f}<<F_{s-c b}$.
} 
absorption phenomenon in thermopile's bolometer, taking into account the average value of bolometer absorbance $a_{\mathrm{s}}$. They also include the average emissitities of different bodies, assuming the hypothesis of greybody behavior for all of them. Finally, thermopile filter transmittance $\chi_{f}(\lambda)$ is taken into account to calculate the flux terms inside filter transmission band for those elements outside the thermopile structure, and filter reflectance $\rho_{f}(\lambda)$ is also considered to work out the flux term radiated by the filter inside and outside the transmission band.

$$
\begin{array}{cc}
\Phi_{g}= & \varepsilon_{g} \cdot a_{s} \int_{0}^{\infty} \chi_{f}(\lambda) \frac{2 h c^{2}}{\lambda^{5} \cdot\left(e^{h c / \lambda K T_{g}}-1\right)} d \lambda \quad \Phi_{p}=\varepsilon_{p} \cdot a_{s} \int_{0}^{\infty} \chi_{f}(\lambda) \frac{2 h c^{2}}{\lambda^{5} \cdot\left(e^{h c / \lambda K T_{p}}-1\right)} d \lambda \\
\Phi_{f}=a_{s} \int_{0}^{\infty}\left(1-\chi_{f}(\lambda)-\rho_{f}(\lambda)\right) \frac{2 h c^{2}}{\lambda^{5} \cdot\left(e^{h c / \lambda K T_{f}}-1\right)} d \lambda & \Phi_{s}=\varepsilon_{s} \int_{0}^{\infty} \frac{2 h c^{2}}{\lambda^{5} \cdot\left(e^{h c / \lambda K T_{s}}-1\right)} d \lambda \\
\Phi_{c c}=\varepsilon_{c c} \cdot a_{s} \int_{0}^{\infty} \frac{2 h c^{2}}{\lambda^{5} \cdot\left(e^{h c / \lambda K T_{c c}}-1\right)} d \lambda \quad \Phi_{c b}=\varepsilon_{c b} \cdot a_{s}^{\infty} \int_{0}^{\infty} \frac{2 h c^{2}}{\lambda^{5} \cdot\left(e^{h c / \lambda K T_{c b}}-1\right)} d \lambda
\end{array}
$$

All these integrals depend on different temperatures: $T_{c b}$ and $T_{p}$ are directly measured using specific RTDs attached to the corresponding bodies. During the GTS calibration process the target temperature $T_{g}$ is measured by a specific contact temperature sensor, while during the normal operation it is the unknown to be measured by the GTS. The temperature of the filter is the same as the temperature of the thermopile's can cap as they are bound by a conductive adhesive $T_{f}=T_{c c}$. And the temperature of the thermopile's can cap is equal to the temperature of the thermopile's can base, $T_{c c}=T_{c b}$, since both are made of the same material (steel) and they are in good thermal contact, except when the in-flight calibration plate is being heated.

Finally, the temperature of the bolometer, $T_{s}$, is obtained from the output voltage of the thermopile [5][8]. The thermopile produces a voltage representation of the temperature difference between its can base (cold junction) and the bolometer (hot junction), equation (5). The GTS thermopiles have 100 thermocouples connected in series and embedded between the 
can and the bolometer, and the term $\left.\alpha_{A B}\right|_{T_{c b}}$ stands for the Seebeck coefficient related to the association of the two materials of the thermopile's thermocouples.

$$
V_{\text {out }}=\left.100 \cdot \alpha_{A B}\right|_{T_{C}} \cdot\left(T_{s}-T_{c b}\right)
$$

The GTS thermal model is completed with the thermopile's thermal gradients estimator. The calibration plate will be heated as part of the in-flight calibration process, generating a thermal gradient in the whole GTS and more specifically in the structure of the thermopile [13], Fig. 3. As shown in Fig. 1, the calibration plate is attached with screws to the thermopiles' hosting piece. Thus, thermal coupling exists between these two pieces and by extension with the thermopiles, based on conduction, through the atmosphere, the screws, and radiation. In this way, a thermal gradient will appear between the thermopiles' can cap at the top and the can base at the bottom. From this, the proposed estimator takes into account the existence of a relationship between the difference in the temperature of the calibration plate before and after heating $T_{p 2^{-}} T_{p 1}$, and the temperature gradient between the thermopile's can base and cap (6). Actually, the assumption of a constant over temperature for the whole can cap during the inflight calibration represents a simplification of the real situation. This relation is assumed to be lineal through the constant $K_{p-c}$ for a certain absolute temperature, since the non linear radiation term can be simplified in a linear term [8].

$$
T_{c c}-T_{c b}=K_{p-c} \cdot\left(T_{p 2}-T_{p 1}\right)
$$

From analysis of equations (4) and (6), we can conclude the need to identify the unknown constants of the sensor model: $K_{l}, K_{2}, K_{3}, \alpha$ and $K_{p-c}$.

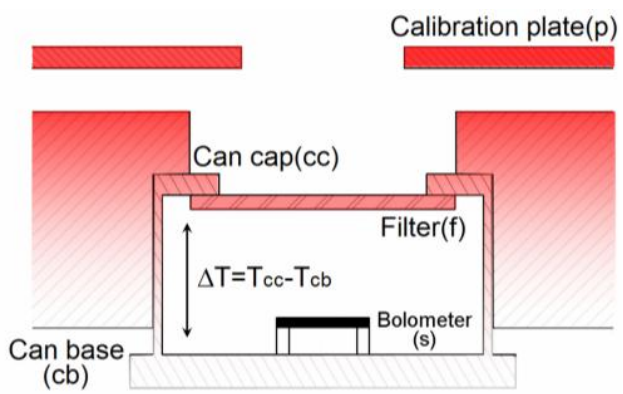

Fig.3 GTS and the thermopile's thermal gradient. 


\section{Experimental setup}

The experimental setup, see Fig. 4a, consists of the MIKRON M315X $(15 \times 15 \mathrm{~cm})$ a large area blackbody calibration source that replaces or simulates the ground surface during the calibration and measuring processes. The GTS, Fig. 5, is located inside the cryostat JANIS JC100 in order to change the thermopile temperature, covering the Martian working range. The thermopile faces the radiation surface of the blackbody through the cryostat IR window made of KRS-5. A mechanical shutter has been inserted between the blackbody and the cryostat to avoid heating the GTS during blackbody temperature stabilization. The output voltage of the thermopiles and the resistance of the different RTDs are measured using a KEITHLEY 2700 multimeter and a KEITHLEY 7700 commutation matrix.

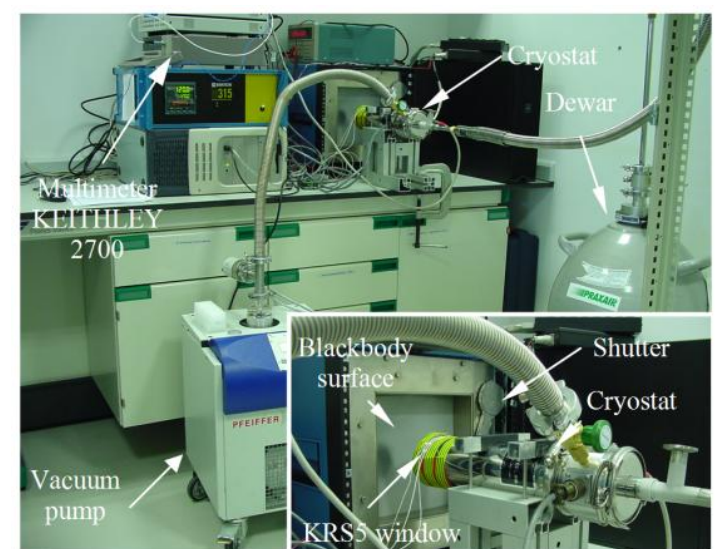

(a)

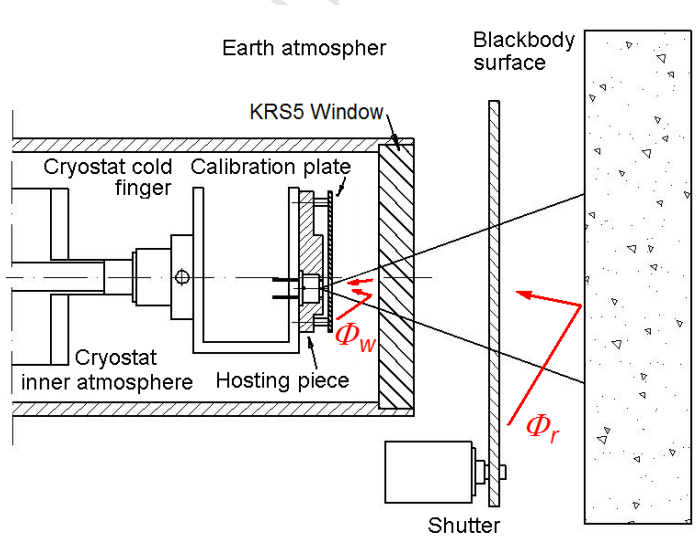

(b)

Fig.4. (a) Experimental setup, (b) Schematic diagram of the setup showing undesired flux terms.

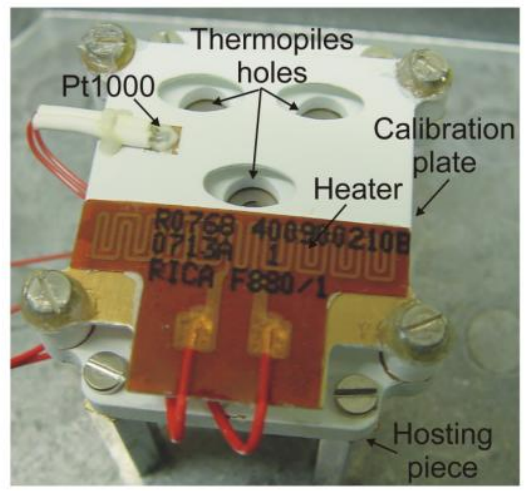

Fig.5 REMS GTS tested model. 


\subsection{Differential equations for REMS GTS model identification}

In order to avoid the uncontrolled but constant heat flux terms derived from the experimental test setup, see Fig. 4, a differential procedure and analysis of the energy balance equation is applied. These undesired terms are: the blackbody environment reflections $\Phi_{r}$, that arise because blackbody emissivity is different from one, and the IR window emissions and reflections, $\Phi_{w}$. These heat flux terms appear in the energy balance equation (3), modifying it until it is converted into (7), where $\chi_{w}$ represents the cryostat window average transmittance.

$$
\alpha \cdot K_{1} \cdot\left(\chi_{w} \cdot \Phi_{g}+\Phi_{w}+\Phi_{r}\right)+(1-\alpha) \cdot K_{1} \cdot \Phi_{p}+K_{1} \cdot \Phi_{f}+\frac{K_{2}}{2} \cdot \Phi_{c c}+\frac{K_{2}}{2} \cdot \Phi_{c b}-\left(K_{1}+K_{2}\right) \cdot \Phi_{s}+K_{3} \cdot\left(T_{c b}-T_{s}\right)=0(7)
$$

The differential procedure combines two energy balance equations (7) for pairs of blackbody or calibration plate temperatures with the same thermopile temperature. Thus, by subtracting the two energy balance equations, in which the temperatures of the cryostat IR window and the laboratory environment remain stable, the unknown but constant heat flux terms, $\Phi_{w}$ and $\Phi_{r}$, are eliminated. A detailed analysis of this procedure is described in each test subsection. Additionally, the differential procedure makes it possible to avoid errors caused by the constant thermal gradients on each thermopile's can, which may appear for each consigned thermopile temperature as the thermopiles' hosting piece is heated or cooled [12].

\section{Tests and results}

This section includes the description and analysis of a series of three tests the objective of which is to identify the sensor model constants $K_{l}, K_{2}, K_{3}, \alpha$ and $K_{p-c}$, that are part of the GTS instrument calibration plan. Additionally, a test designed to show ability to measure the temperature of the target is also included.

The results shown in the following subsections have been obtained from a GTS engineering model constructed for test purposes and a thermopile with a band pass filter of 8 to $14 \mu \mathrm{m}$, see Electronic Annex 1 in the online version of this article. Table 1 summarises the test conditions and the temperatures consigned to the different GTS elements. In the case of the identification tests, sampled variables are only collected after the consigned temperatures are stable. 
Table 1 Test summary of experimental temperature references and test conditions

\begin{tabular}{ccccc}
\hline Test & $\begin{array}{c}\text { Blackbody } \\
\text { temperature } \\
\boldsymbol{T}_{\boldsymbol{g}}\end{array}$ & $\begin{array}{c}\text { Thermopile } \\
\text { temperature } \\
\boldsymbol{T}_{\boldsymbol{c}}\end{array}$ & $\begin{array}{c}\text { Calibration plate } \\
\text { temperature } \\
\boldsymbol{T}_{\boldsymbol{p}}\end{array}$ & $\begin{array}{c}\text { Cryostat } \\
\text { atmosphere }\end{array}$ \\
\hline Identification of $K s$ & $\sim[40,60, \ldots 120]^{\circ} \mathrm{C}$ & $\sim[-10,0,10, \ldots 40]^{\circ} \mathrm{C}$ & - & Vacuum \\
\hline Identification of $\alpha$ & $\sim[40,60, \ldots 120]^{\circ} \mathrm{C}$ & $\sim 40^{\circ} \mathrm{C}$ & $\sim 40^{\circ} \mathrm{C}$ & $\begin{array}{c}\text { Earth } \\
\text { atmosphere }\end{array}$ \\
\hline $\begin{array}{c}\text { Identification of } \\
\text { thermopile thermal } \\
\text { gradient } K_{p-c}\end{array}$ & $\sim 23^{\circ} \mathrm{C}$ & $\sim 23-28^{\circ} \mathrm{C}$ & $\sim T_{c b}+[0,9,13,18]^{\circ} \mathrm{C}$ & $\begin{array}{c}\text { Earth } \\
\text { atmosphere }\end{array}$ \\
\hline $\begin{array}{c}\text { Test of sensor } \\
\text { performance }\end{array}$ & $\sim 23^{\circ} \mathrm{C}$ & $\sim 23^{\circ} \mathrm{C}$ & $\sim T_{c b}+[0,11]^{\circ} \mathrm{C}$ & $\begin{array}{c}\text { Earth } \\
\text { atmosphere }\end{array}$ \\
\hline
\end{tabular}

Table 2 summarizes the average practical value for the emissivities of the different bodies, and for the filters transmittance and reflectance, under the hypothesis of greybody behaviour. Blackbody and calibration plate emissivities, $\varepsilon_{g}$ and $\varepsilon_{p}$, are computed from spectral data of the corresponding surfaces, taking into account the form of Plank's law for the tested temperatures. From spectral data, it can also be said that the cryostat KRS-5 window $\chi_{w}$ and the thermopile window $\chi_{f}(\lambda)$ present a plain spectral transmittance inside the band-pass. Bolometer absorptivity $a_{s}$ and emissivity $\varepsilon_{s}$ are vey high, since it is cover with a high emissivity coating (Ag-black), but its exact value has not been tested. As approximation, it is assumed that both are equal to 1 .

Finally, we have the emissivities of the inner side of the thermopile's can, $\varepsilon_{c c}$ and $\varepsilon_{c b}$, and the thermopile's filter inner side reflectance, $\rho_{f}(\lambda)$. IR filters like the one of the thermopile present high reflectance outside the band pass, while in the band pass the reflectance is very low. Additionally, the filter diameter $(2 \mathrm{~mm})$ is relatively small versus the thermopile's can diameter (8mm). So, the thermopile's can and the filter form almost a closed structure. In addition to that, both elements have the same or quite similar temperature. Therefore, considering the internal reflections inside the thermopile's can, the whole structure behaves as a cavity blackbody, which is supposed to have a very high emissivity. As a result of the former, filter reflectance is assumed to have a null value, while thermopile's can emissivity is approximated to 1 .

Table 2 Practical data required for heat flux terms calculations

\begin{tabular}{cc}
\hline Variable & Value \\
\hline$\varepsilon_{g}$ & 0.9433 \\
\hline$\varepsilon_{p}$ & 0.9018 \\
\hline$\varepsilon_{c c}$ & 1 \\
\hline$\varepsilon_{c b}$ & 1 \\
\hline
\end{tabular}




\begin{tabular}{cc}
\hline$\varepsilon_{s}=a_{s}$ & 1 \\
\hline$\chi_{w}$ & 0.7566 from 0.6 to $40 \mu \mathrm{m}$ \\
\hline$\chi_{f}(\lambda)$ & 0.754 from 8 to $14 \mu \mathrm{m}$, otherwise 0 \\
\hline$\rho_{f}(\lambda)$ & 0 \\
\hline
\end{tabular}

\subsection{Identification test for $K_{1}, K_{2}$ and $K_{3}$}

To carry out this first test the calibration plate has been removed, ensuring that $\alpha=1$. In addition, because the test is carried out in homogeneous temperature conditions without heating up the calibration plate, the temperature in the thermopile base, cap and filter are equal, $T_{c c}=T_{c b}=T_{f}$. Nevertheless, if there is any thermopile spatial thermal gradient its effect is avoided because of the differential analysis. From these premises the energy balance equation (7) can be rewritten,

$$
K_{1} \cdot\left(\chi_{w} \cdot \Phi_{g}+\Phi_{w}+\Phi_{r}\right)+K_{1} \cdot \Phi_{f}+\frac{K_{2}}{2} \cdot \Phi_{c c}+\frac{K_{2}}{2} \cdot \Phi_{c b}-\left(K_{1}+K_{2}\right) \cdot \Phi_{s}+K_{3} \cdot\left(T_{c b}-T_{s}\right)=0
$$

From equation (8) and for the pairs of blackbody temperatures in table 1, with the same or fairly similar thermopile temperature, as from a practical point of view keeping the thermopile at exactly the same temperature is impossible, the differential analysis results in,

$$
K_{1} \cdot\left(\chi_{w} \cdot \Phi_{g 1}-\chi_{w} \cdot \Phi_{g 2}\right)+K_{1} \cdot\left(\Phi_{f 1}-\Phi_{f 2}\right)+\frac{K_{2}}{2} \cdot\left(\Phi_{c c 1}-\Phi_{c c 2}\right)+\frac{K_{2}}{2} \cdot\left(\Phi_{c b 1}-\Phi_{c b 2}\right)-\left(K_{1}+K_{2}\right) \cdot\left(\Phi_{s 1}-\Phi_{s 2}\right)+K_{3} \cdot\left(T_{c b 1}-T_{c b 2}-T_{s 1}+T_{s 2}\right)=0
$$

The identification of $K_{1}, K_{2}$ and $K_{3}$ is achieved based on equation (9) and a linear least square algorithm that tries to minimize the estimation error. To carry out the algorithm it is necessary to know the calculations of the heat flux terms based on (4) and the temperature measurements of the whole set of thermopile and blackbody temperature references in table 1. As a result, assuming that the value of $K_{1}=1$, the relative values of the constants $K_{2}$ and $K_{3}$, are obtained. Table 3 summarizes the results of the process, including the root mean square error associated with the estimation of blackbody temperatures, while Fig. 6 graphically presents specific results.

\begin{tabular}{|c|c|c|}
\hline Variable & Value & Target temperature error ${ }_{\mathrm{RMS}}$ \\
\hline$K_{1}$ & $1 \mathrm{~m}^{2}$ & \\
\hline$K_{2}$ & $23.6095 \mathrm{~m}^{2}$ & $0.0529^{\circ} \mathrm{C}$ \\
\hline$K_{3}$ & $133.0812 \mathrm{~W} / \mathrm{K}$ & \\
\hline$\alpha$ & 0.6605 & $0.0287^{\circ} \mathrm{C}$ \\
\hline
\end{tabular}

Table 3 Calibration test results 


\begin{tabular}{ll}
\hline$K_{p-c}$ & 0.0012136 \\
\hline
\end{tabular}

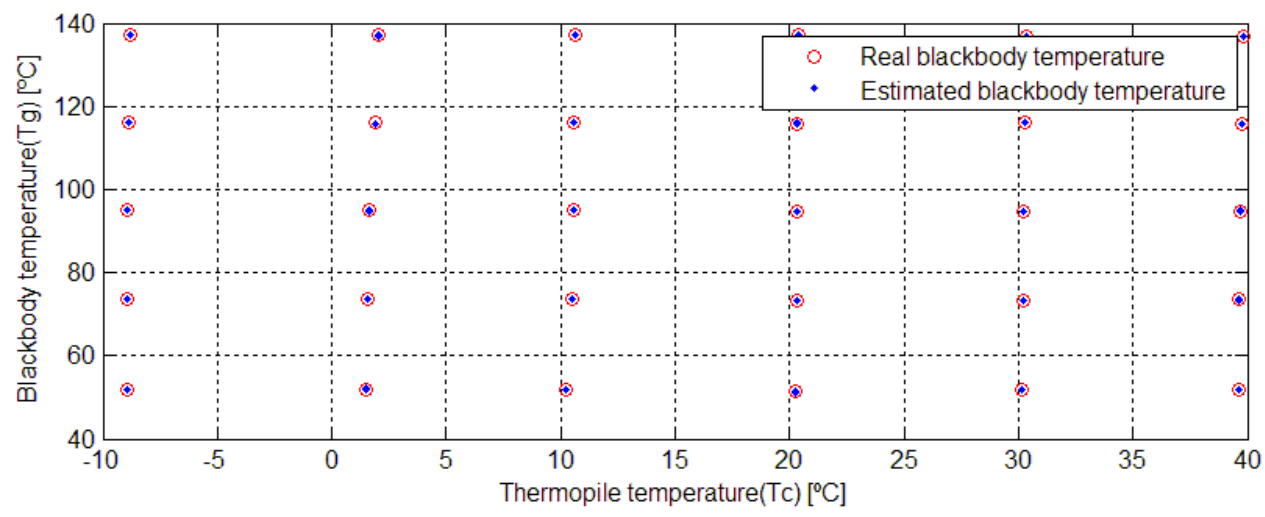

Fig. 6 Blackbody temperature estimation associated with the identification of $K_{1}, K_{2}$ and $K_{3}$.

\subsection{Test for identification of $\alpha$}

In this test the calibration plate is mounted on the front of the thermopile and the values of the previously identified constants $K_{l}, K_{2}$ and $K_{3}$ are required. The test consists of setting different temperatures for the blackbody, table 1 . As the temperature of both the thermopile and the calibration plate are stable the temperature in the thermopile base, cap and filter are equal, $T_{c c}=T_{c b}=T_{f}$. A differential analysis of the energy balance equation for two different blackbody temperatures can again be applied to (7),

$\alpha \cdot K_{1} \cdot\left(\chi_{w} \cdot \Phi_{g 1}-\chi_{w} \cdot \Phi_{g 2}\right)+(1-\alpha) \cdot K_{1} \cdot\left(\Phi_{p 1}-\Phi_{p 2}\right)+K_{1} \cdot\left(\Phi_{f 1}-\Phi_{f 2}\right)+\frac{K_{2}}{2} \cdot\left(\Phi_{c c 1}-\Phi_{c 2}\right)+\frac{K_{2}}{2} \cdot\left(\Phi_{c b 1}-\Phi_{c b 2}\right)-\left(K_{1}+K_{2}\right) \cdot\left(\Phi_{s 1}-\Phi_{s 2}\right)+K_{3} \cdot\left(T_{c b 1}-T_{c b 2}-T_{s 1}+T_{s 2}\right)=0$

and finally equation (10) can be solved for $\alpha$,

$\alpha=\frac{-K_{1} \cdot\left(\Phi_{p 1}-\Phi_{p 2}\right)-K_{1} \cdot\left(\Phi_{f 1}-\Phi_{f 2}\right)-\frac{K_{2}}{2} \cdot\left(\Phi_{c c 1}-\Phi_{c c 2}\right)-\frac{K_{2}}{2} \cdot\left(\Phi_{c b 1}-\Phi_{c b 2}\right)+\left(K_{1}+K_{2}\right) \cdot\left(\Phi_{s 1}-\Phi_{s 2}\right)-K_{3} \cdot\left(T_{c b 1}-T_{c b 2}-T_{s 1}+T_{s 2}\right)}{K_{1} \cdot\left(\chi_{w} \cdot \Phi_{g 1}-\chi_{w} \cdot \Phi_{g 2}\right)-K_{1} \cdot\left(\Phi_{p 1}-\Phi_{p 2}\right)}$

The individual and average values of $\alpha$ as a result of applying equation (11) to the set of temperature references of table 1 are shown in Fig. 7. Table 3 summarizes the average value of $\alpha$ and the associated root mean square error in the estimation of blackbody temperature. 


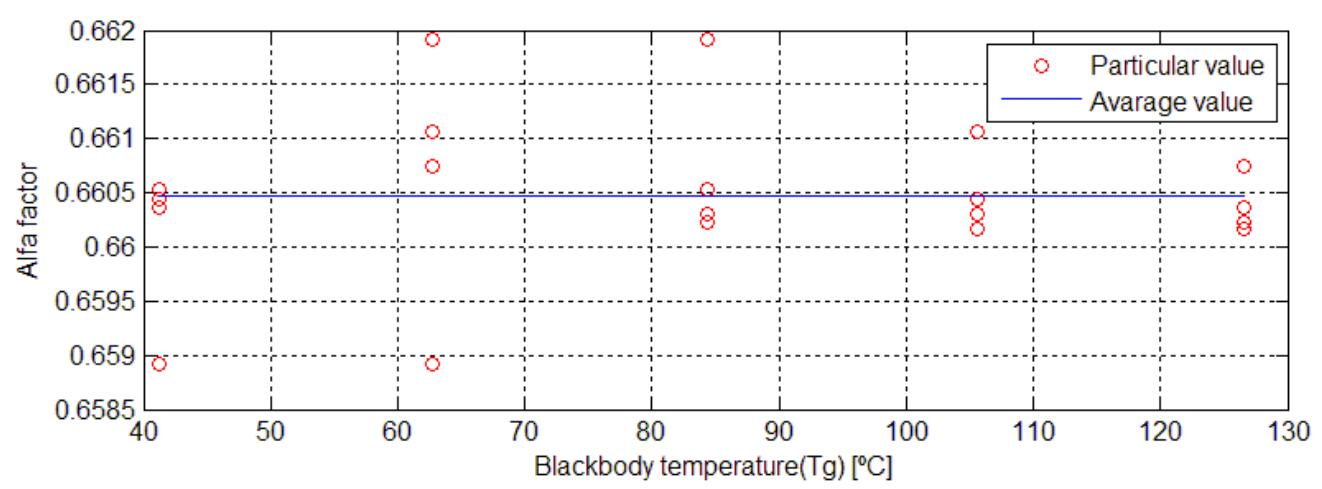

Fig.7 Experimental data for ground relative FOV $\alpha$, as a function of $T_{g}$.

\subsection{Test for identification of thermopile thermal gradient constant $K_{p-c}$}

This test is designed to establish a relationship between the increase in the temperature of the calibration plate and the thermal gradient generated between the thermopile's can cap and base, $T_{c c}-T_{c b}$. In the case of this test, window reflections cannot be avoided by using a differential analysis of the energy balance equation as the differential analysis is developed for different calibration plate temperatures and so the reflections are different for each test point. Thus, the setup of the test has been slightly modified, removing the cryostat window $\chi_{w}=1$, in order to eliminate these window reflections.

The test consists mainly of setting different temperatures for the calibration plate, using for this the associated heater. While the temperature of the blackbody remains constant, the temperature of the thermopile changes freely due to the heat coming from the calibration plate. The aim of this procedure is to emulate the appearance of thermal gradients on the thermopile's can due to the in-flight calibration algorithm running during Martian operations.

Once again, from the energy balance equation (7), in which the value of the constants $K_{1}, K_{2}$, $K_{3}$ and $\alpha$ have previously been identified, a differential analysis is applied in order to avoid the error term $\Phi_{r}$. Equation (12) represents the result of the differential procedure for two different calibration plate temperatures. The first temperature, in which the calibration plate is not heated, is used as a reference point and so it is assumed that $T_{c c l}=T_{f l}=T_{c b l}$. In this way equation (12) 
can be solved for the unknown temperature $T_{c c 2}$, which is equal to $T_{f 2}$, from the value of $\Phi_{c c 2}$ and $\Phi_{f 2}$ using numerical methods. The other temperatures and heat flux terms are all known. $(1-\alpha) \cdot K_{1} \cdot\left(\Phi_{p 1}-\Phi_{p 2}\right)+K_{1} \cdot\left(\Phi_{f 1}-\Phi_{f 2}\right)+\frac{K_{2}}{2} \cdot\left(\Phi_{c c 1}-\Phi_{c c 2}\right)+\frac{K_{2}}{2} \cdot\left(\Phi_{c b 1}-\Phi_{c b 2}\right)-\left(K_{1}+K_{2}\right) \cdot\left(\Phi_{s 1}-\Phi_{s 2}\right)+K_{3} \cdot\left(T_{c b 1}-T_{c b 2}-T_{s 1}+T_{s 2}\right)=0$

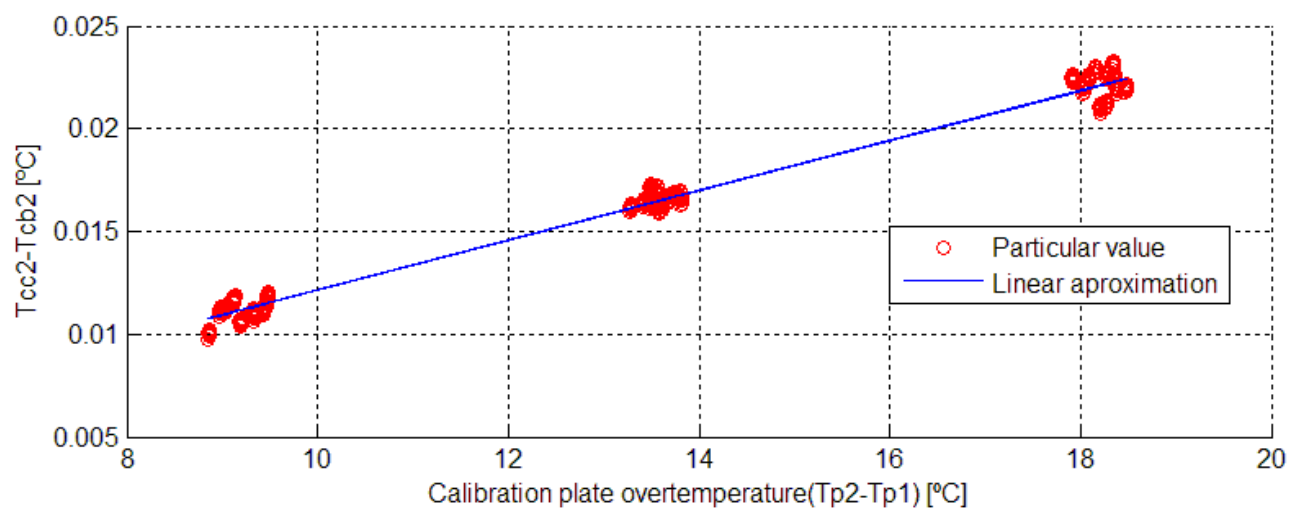

Fig. 8 Experimental data for thermopile thermal gradients constant estimation $K_{p-c}$.

The result of applying equation (12) to the set of temperatures in table 1, taking several pieces of data for each calibration plate temperature is shown in Fig.8. The figure shows the linear regression (6) between the calibration plate over-temperature and the thermopile thermal gradients through the constant $K_{p-c}$, the value of which is shown in table 3. As it demonstrates, practical data validate the lineal model of the thermal gradient (6). Despite the relatively small value of the thermopile thermal gradient, which is between $0.01-0.023^{\circ} \mathrm{C}$, its relevance in target temperature determination is important as principally it modifies the heat term $\frac{K_{2}}{2} \cdot \Phi_{c c}$, which has a high weighting in the energy balance equation.

\subsection{Test of sensor performance}

This final test, which is not part of the instrument calibration plan, is designed to analyse the behaviour of the proposed model in the estimation of blackbody temperature, taking into account the identified values of model constants. The test aims to recreate the temperature measurement process on Mars and in this way its setup is shared with the previous test, 
removing the cryostat window. The test, see Fig. 9, consists of sampling GTS temperature variables for 100 minutes with a sampling period of 1 minute, after 30 minutes the temperature of the calibration plate is increased using the associated heater in order to simulate the running of the in-flight calibration algorithm, while the temperature of the thermopiles evolves freely, table 1.

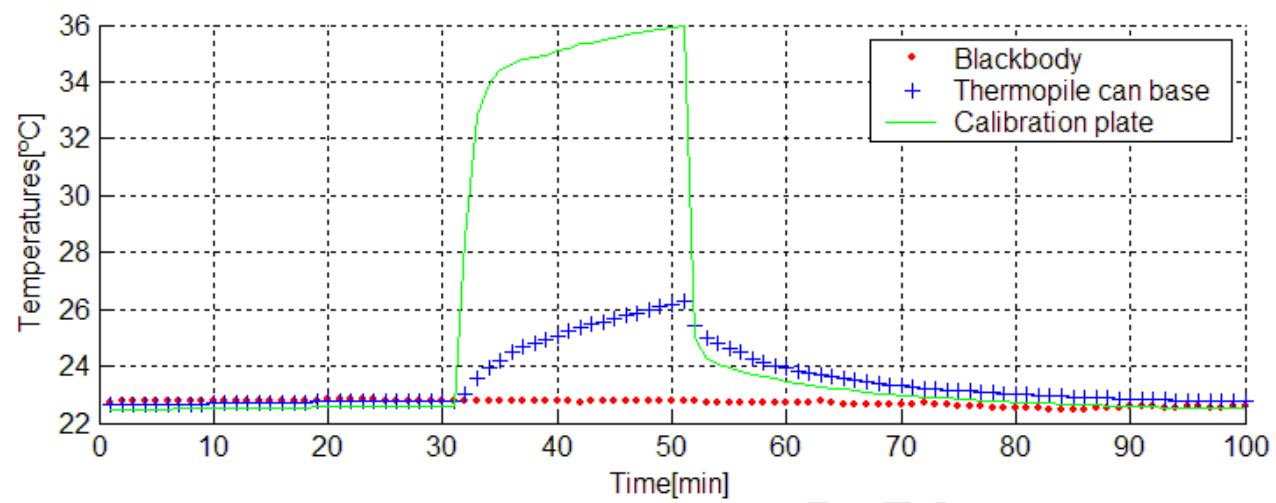

Fig. 9 Evolution of temperatures during the sensor performance test.

In order to estimate the temperature of the blackbody, energy balance equation (7) must be solved for $\Phi_{g}(13)$ and after that by using numerical methods for $T_{g}$. This procedure is not differential, and in order to avoid the errors caused by the emissivity of the blackbody that differs from one $\varepsilon_{g} \neq 1$ and reflections from the environment, the selected temperature of the blackbody is equal to the temperature of the environment, thus the blackbody's emissivity can be assumed to be $\varepsilon_{g}=1$. Additionally, the cryostat window has been newly removed in order to eliminate window influence $\Phi_{w}$, which means that $\chi_{w}=1$. To correctly estimate the blackbody's temperature while the calibration plate is being heated, equations (6) and (7), means a reference value for $T_{p}$ must be taken so the calibration plate over-temperature can be determined and from it the value of $T_{c c}$ and $T_{f}$. The reference has been established at the $30^{\text {th }}$ minute.

$$
\Phi_{g}=\frac{(1-\alpha) \cdot K_{1} \cdot \Phi_{p}+K_{1} \cdot \Phi_{f}+\frac{K_{2}}{2} \cdot \Phi_{c c}+\frac{K_{2}}{2} \cdot \Phi_{c b}-\left(K_{1}+K_{2}\right) \cdot \Phi_{s}+K_{3} \cdot\left(T_{c b}-T_{s}\right)}{\alpha \cdot K_{1}}
$$

where $T_{c c}=T_{f}=K_{p-c} \cdot\left(T_{p}-T_{p, 30 \min }\right)+T_{c b}$ 
The results of applying equation (14) for the whole set of data are shown in Fig. 10, where the real and estimated blackbody temperatures are plotted. Data present a root mean square error of $0.1032^{\circ} \mathrm{C}$, which is within the calibration boundary error of the RTDs used in the test and is compliant with REMS GTS requirements. For a few minutes after the process of heating and cooling the calibration plate has started the estimation error is higher. This is due to the temporal thermal gradient of the thermopile's can [12], which as temperatures have not reached a stable state, the calibrated value of $K_{p-c}$ cannot be assumed.

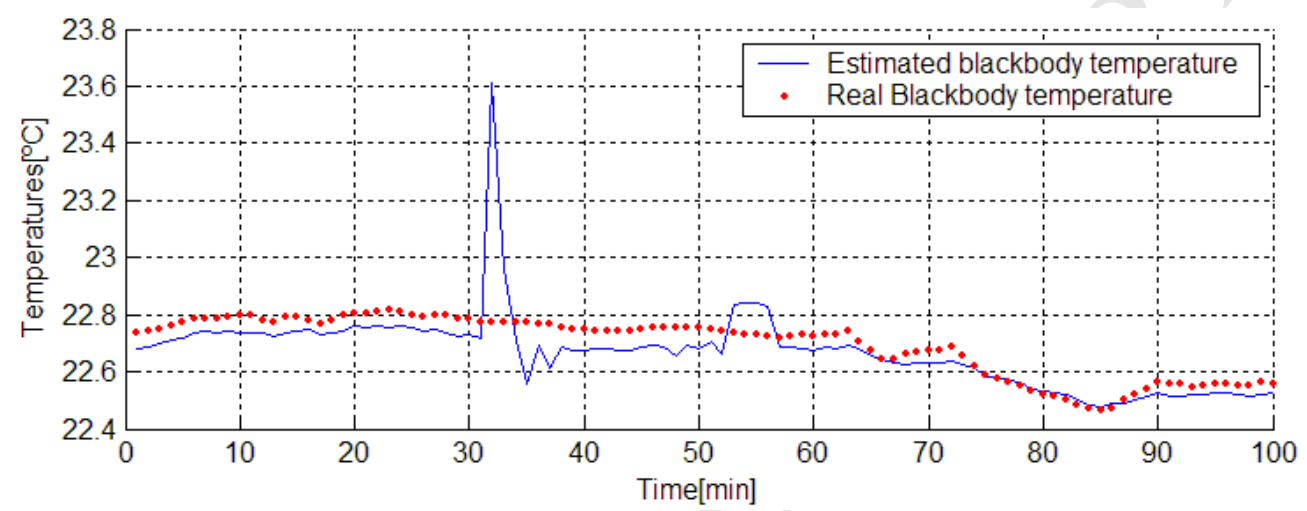

Fig. 10 Blackbody temperature estimation.

\section{Conclusions and future work}

This paper proposes a new simplified mathematical model for the REMS GTS sensor, which can be applied to similar pyrometers. Unlike simpler models, this one is based on the thermal and electrical operation of the thermopile and the physical structure of the sensor. To demonstrate the performance of the model, practical results are provided with the aim of showing: Firstly, the validity of the proposed model and methods for characterising the REMS GTS, due to the low least-squares error presented during the different calibration tests, and secondly, the capability of measuring the temperature of a target with a root mean square error below $0.15^{\circ} \mathrm{C}$, compliant with sensor specifications.

Despite the relatively simple model proposed, the actual physical situation is more complicated. In the identification of $K_{1}, K_{2}$ and $K_{3}$, aspects such as, thermopile's can thermal model, thermopile filter transmittance variation versus temperature and the appearance of inner temperature gradients in thermopile elements may warrant additional consideration being given 
to sensor model variation versus temperature and the calibration set-up. This implies that the identification test for these constants must be run covering the Martian REMS GTS working temperature range, and the values of these constants may be different for different temperature ranges within this working range.

Despite the fact that the identified value of $K_{p-c}$ is constant and depends on the thermal coupling between the calibration plate and the thermopile's hosting piece, it may also depend on the thermal coupling between the entire GTS and its support structure. In addition, atmospheric conditions and the absolute temperature may also be important to the value of $K_{p-c}$, as they modify thermal coupling through atmosphere conduction and radiation. Once again, to sum up we could say that the $K_{p-c}$ constant of the REMS GTS flight model must be calibrated with the GTS located in its end position inside a test chamber filled with a Martian like atmosphere and taking into account the absolute temperature working range. Depending on the absolute temperature, this may imply obtaining different values for $K_{p-c}$.

\section{Acknowledgements}

This work forms part of the research project regarding the design and scientific issues of the NASA/MSL REMS. The authors would like to express special thanks to all REMS Co-Is, collaborators and the INTA Metrological department, who participate in a variety of ways to the development of REMS GTS, as well as the referees and their fruitful contributions. Also acknowledge the financial support provided by the Spanish institutions CYCIT through the ESP2007-65862 grant and CDTI.

\section{References}

[1] G. Pezzotti, P. Coppa, F. Liberati, Low radiation pyrometer for measuring forehead skin temperature, Revista facultad de ingeniería 38 (2006) 128-135.

[2] C. Escriba, E. Campo, D. Estève, J.Y. Fourniols, Complete analytical modelling and analysis of micro-machined thermoelectric uncooled IR sensors, Sensor and actuators A 120 (2005) 267-276. 
[3] T Elbel, S poser, H. Fischer, Thermoelectric radiation micro-sensors, Sensors and actuators A, 41-42 (1994) 493-496

[4] J.E. Muller, E.Kessler, U. Dillner, P. Ratz, K.D. Stock J. Metzdorf, Large highly sensitive thin-film thermopiles as radiometric detector standard. Micro-technology in metrology, and metrology in Microsystems, 31(8) (2000) 147-148.

[5] A. Grafa, M.Arndta,G. Gerlachb. Seebeck's affect of micro-machined thermopiles on infrared detection. A review. (eds.) Estonian Acad. Sci. Eng., 13(4), 338-353, 2007.

[6] www.desxterResearch.com Miniature amplifier PCB

[7] J.P. Ploteau, P. Glouannec, H. Noel, Conception of thermoelectric flux meters for infrared radiation measurements in industrial furnaces. Applied Thermal Engineering 27, 674-681(2007) [8] M.C. Foote, Temperature stabilization requirements for unchopped thermal detectors, Proc. of SPIE conference on infrared technology and applications XXV, vol. 3698, (1999).

[9] http://mars.jpl.nasa.gov/msl Mars Science Laboratory

[10] www.ipht-jena.de IPHT web page.

[11] F.P. Incropera, D.P. Dewit, Introduction to Heat Transfer, Wiley, New York, 1985.

[12] M. Liess, A. Charlebois, M. Hausner, H. Ernst, H. Kragözoglu, J. Schilz, Stabilization of the output signal of thermopile sensors in the thermal environment of automotive applications, Proc. of SPIE Photonic Applications for Aerospace, Transportation and Harsh Environments, vol. 6379, (2006).

[13]L. Boidens, 2008. International patent WO 2008/139321 A1, Infrared Radiometer.

[14] J. Gomez-Elvira, L. Castañer, A. Lepinette, J. Moreno, J. Polko, E. Sebastián, J. Torres, M.P. Zorzano and REMS team, REMS, an instrument for Mars Science laboratory Rover, Lunar and Planetary Science XXXX, (2009). 


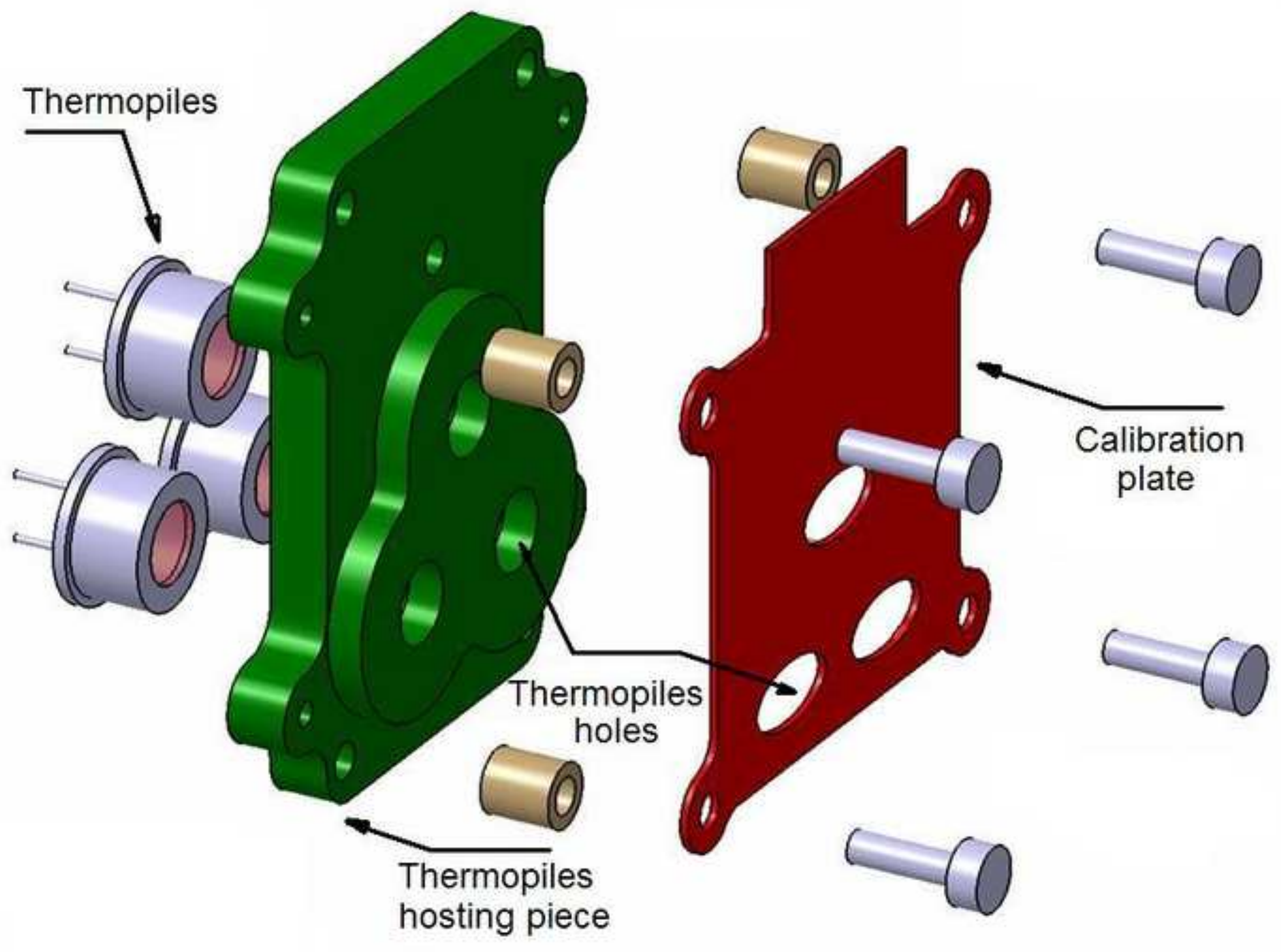
hosting piece 

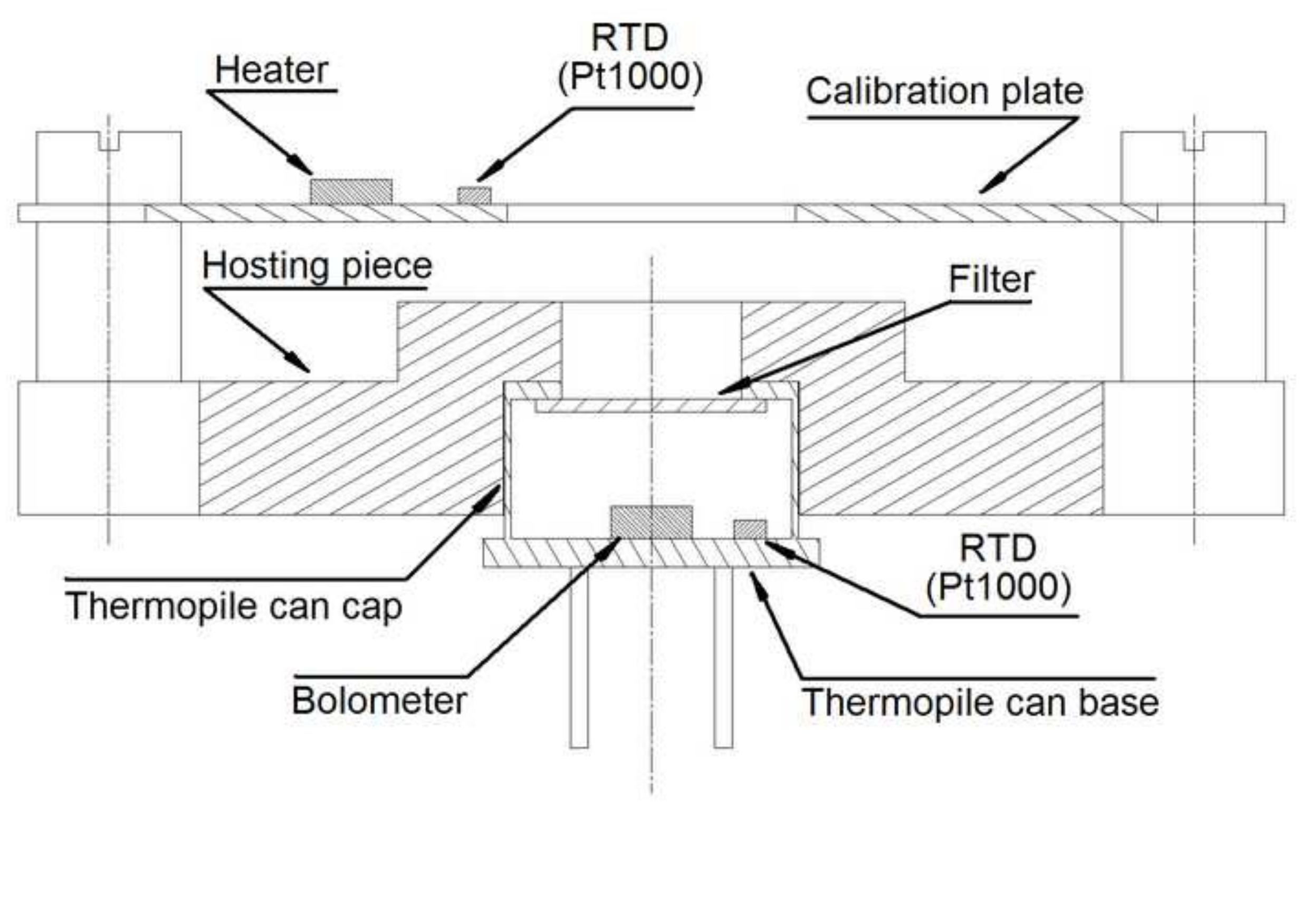


\section{Ground(g)}

\section{Calibration plate $(p)$}

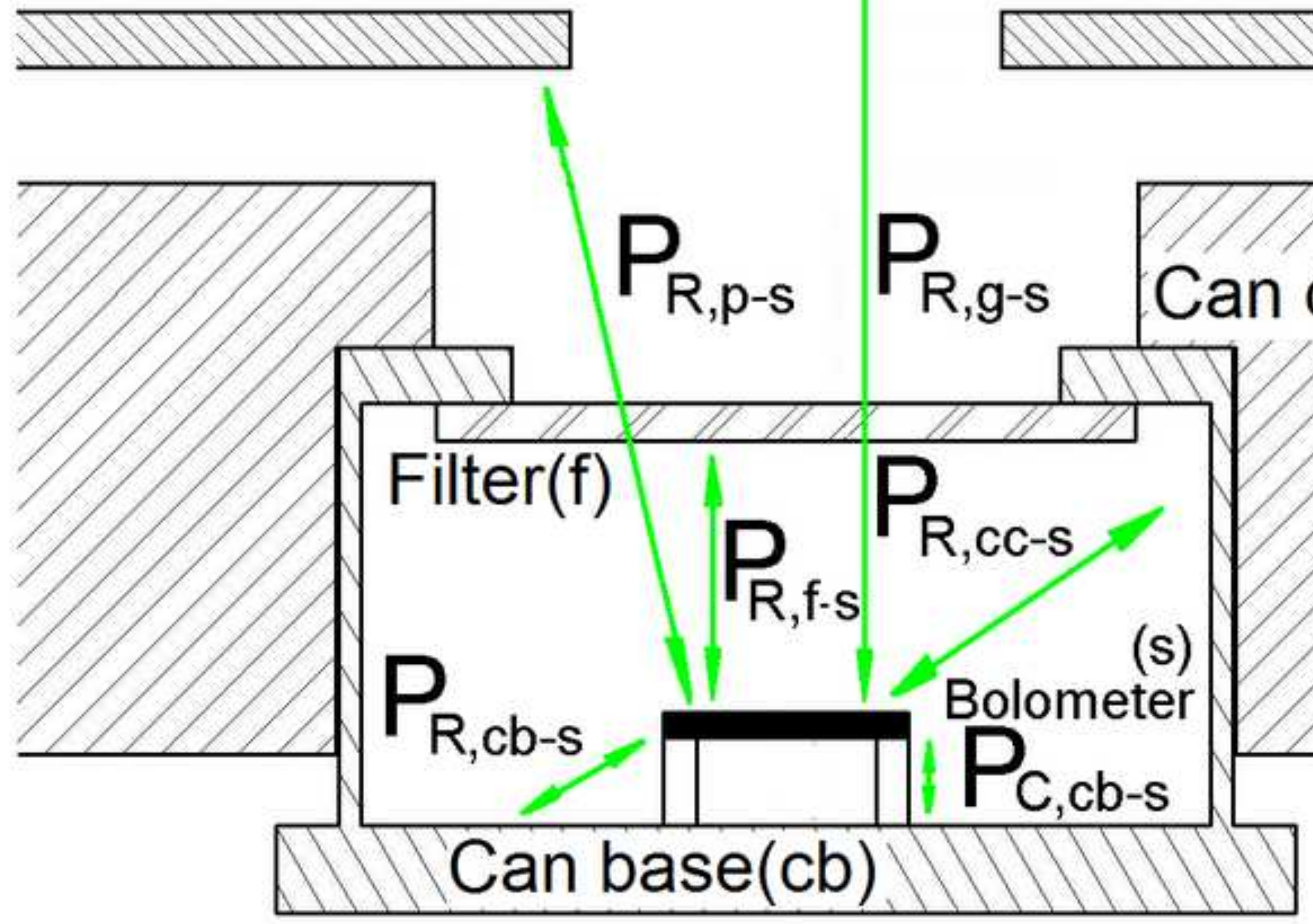

Can base(cb) 


\section{Ground(g)}
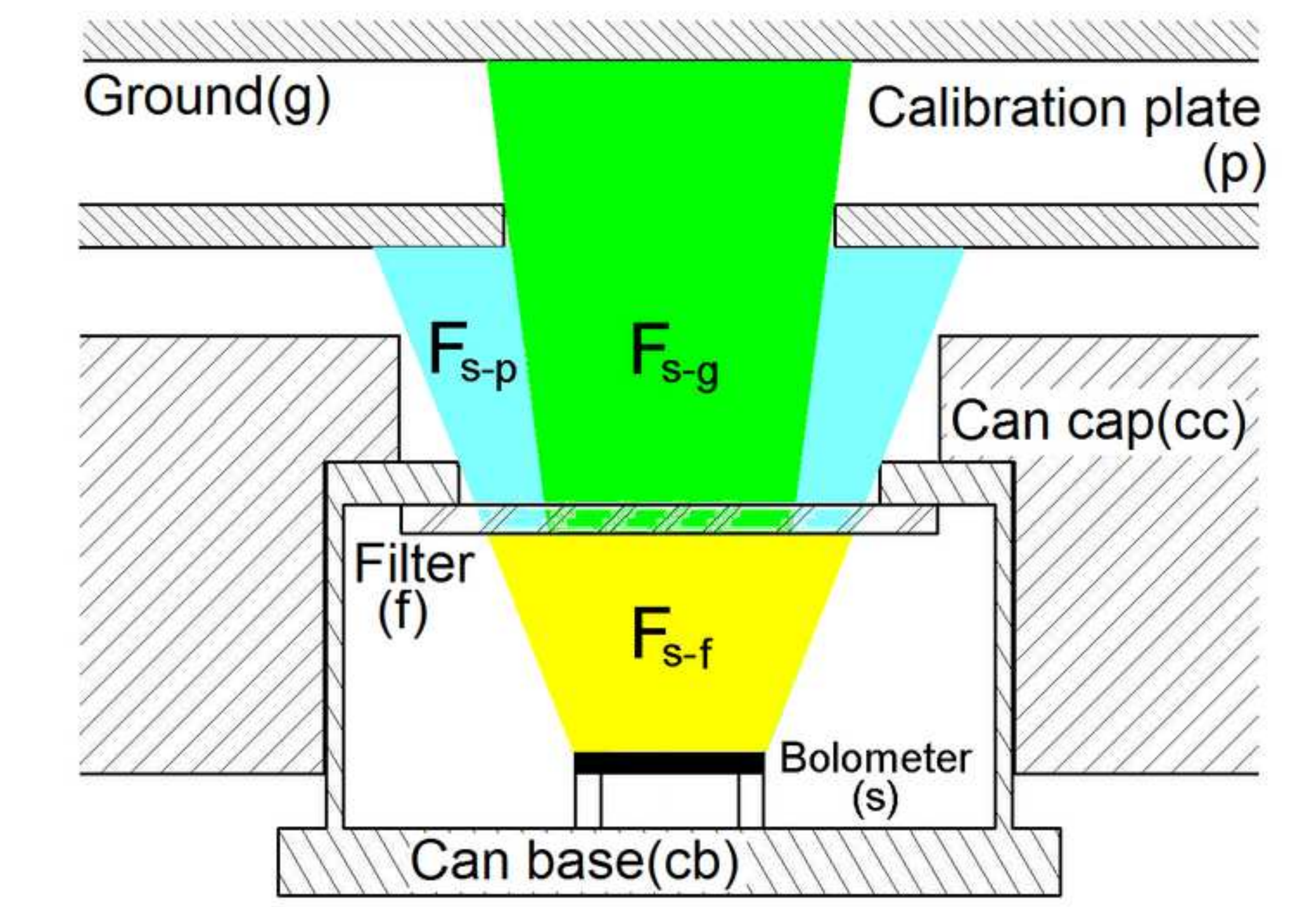

(1)

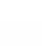

\begin{tabular}{ll|}
$(\mathrm{f}) \quad \mathrm{F}_{\mathrm{s}-\mathrm{f}}$ \\
Can base(cb) \\
Calometer \\
$(\mathrm{s})$
\end{tabular}

Can cap(cc) (1)

(1)

.

.
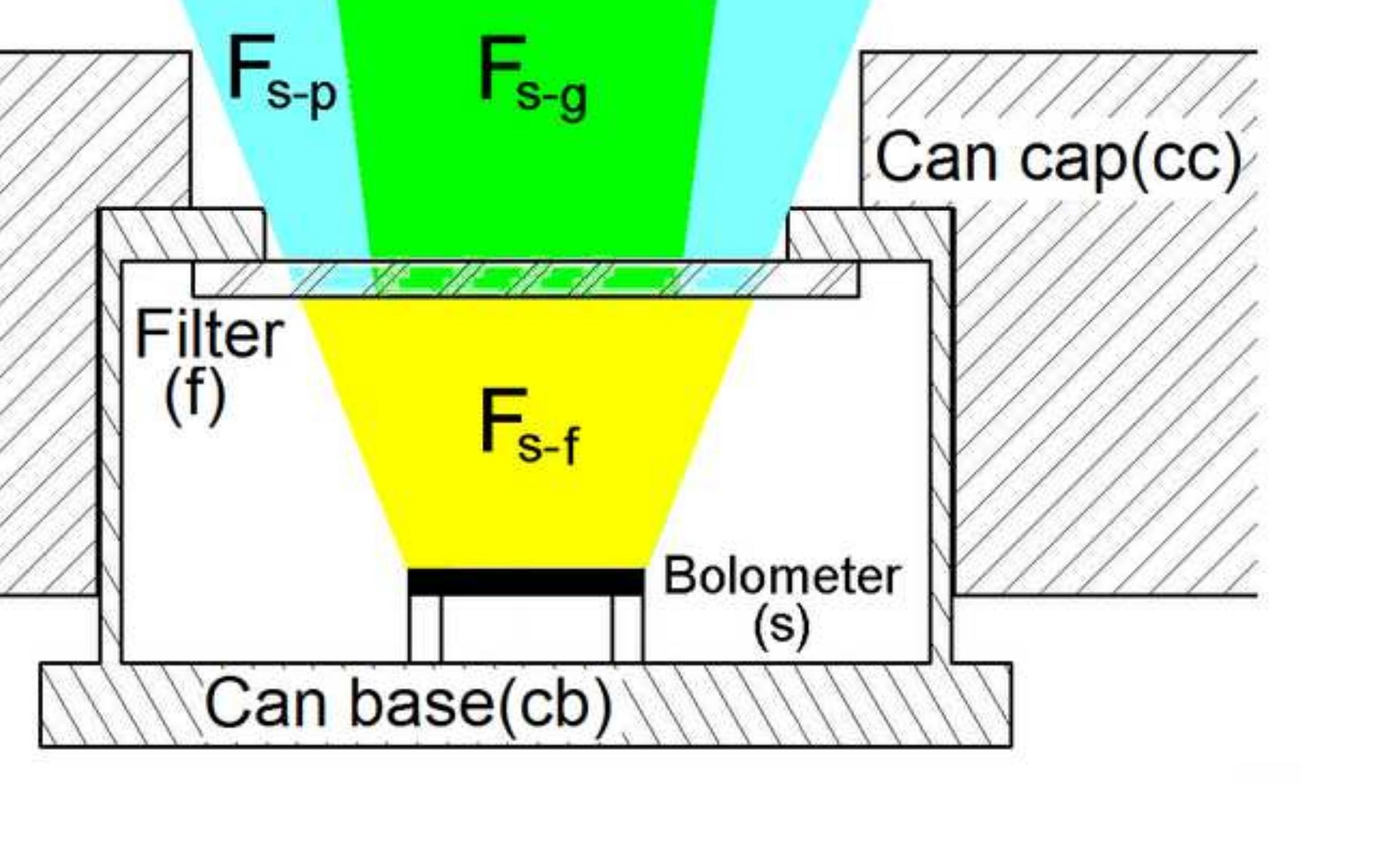
Calibration plate $(p)$

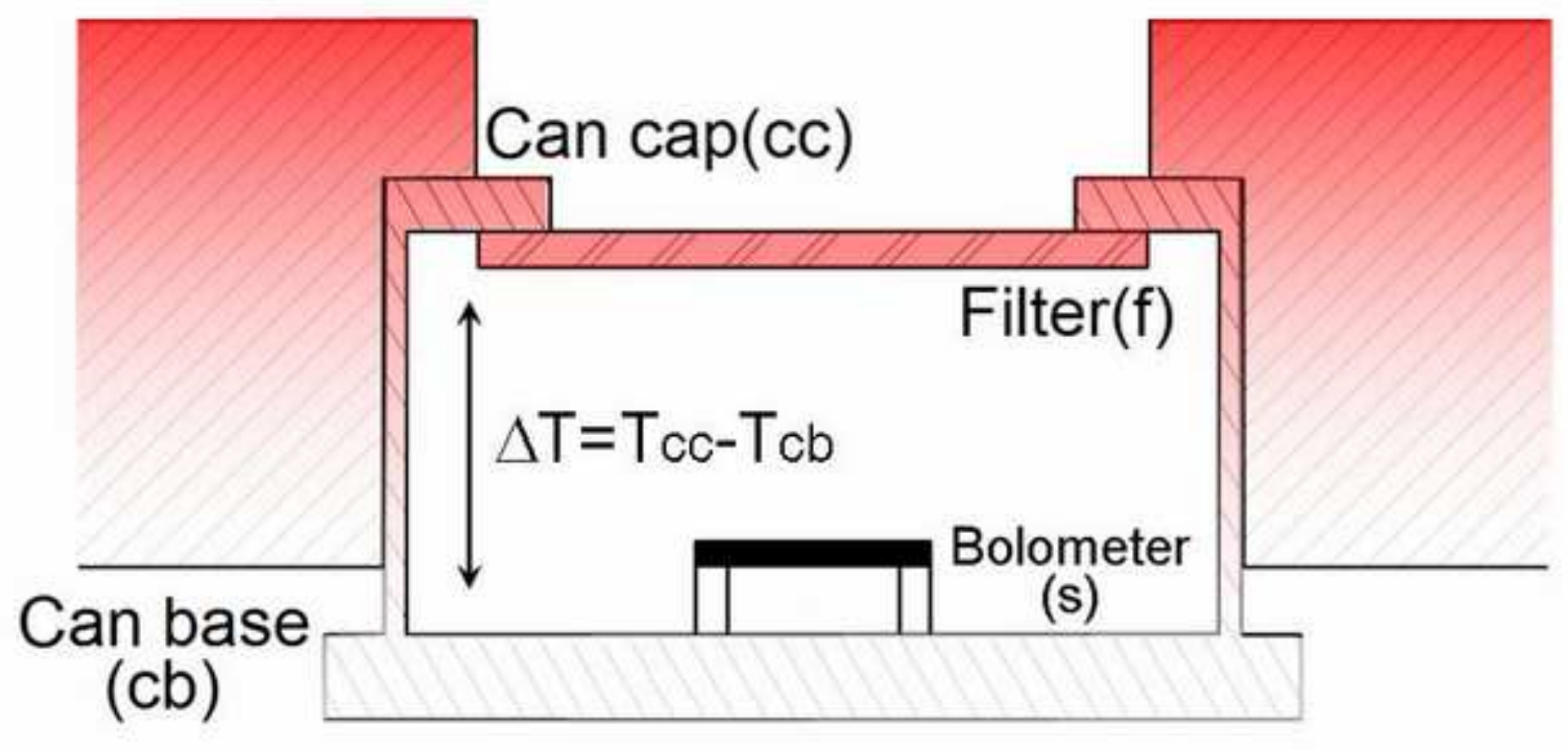




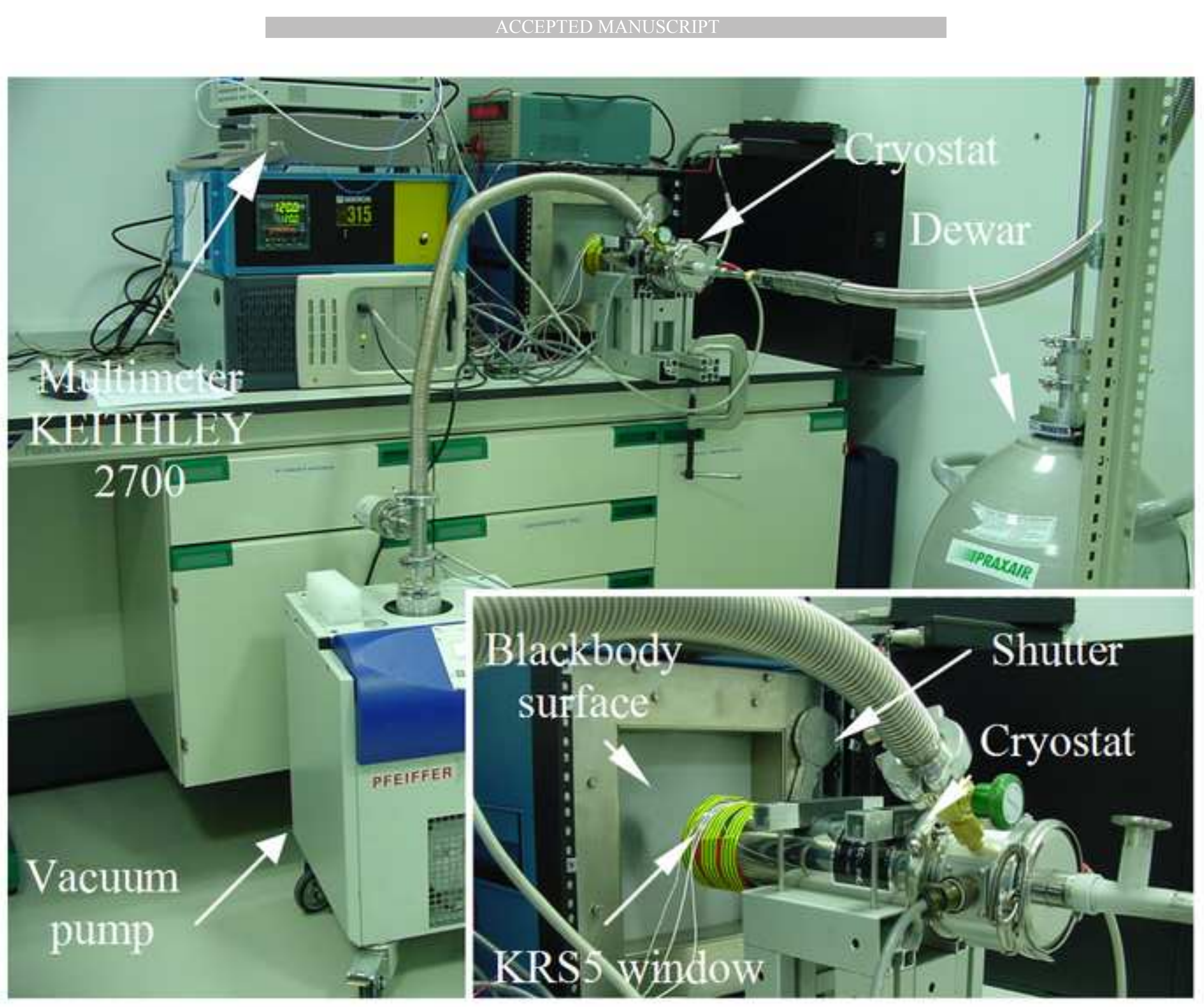




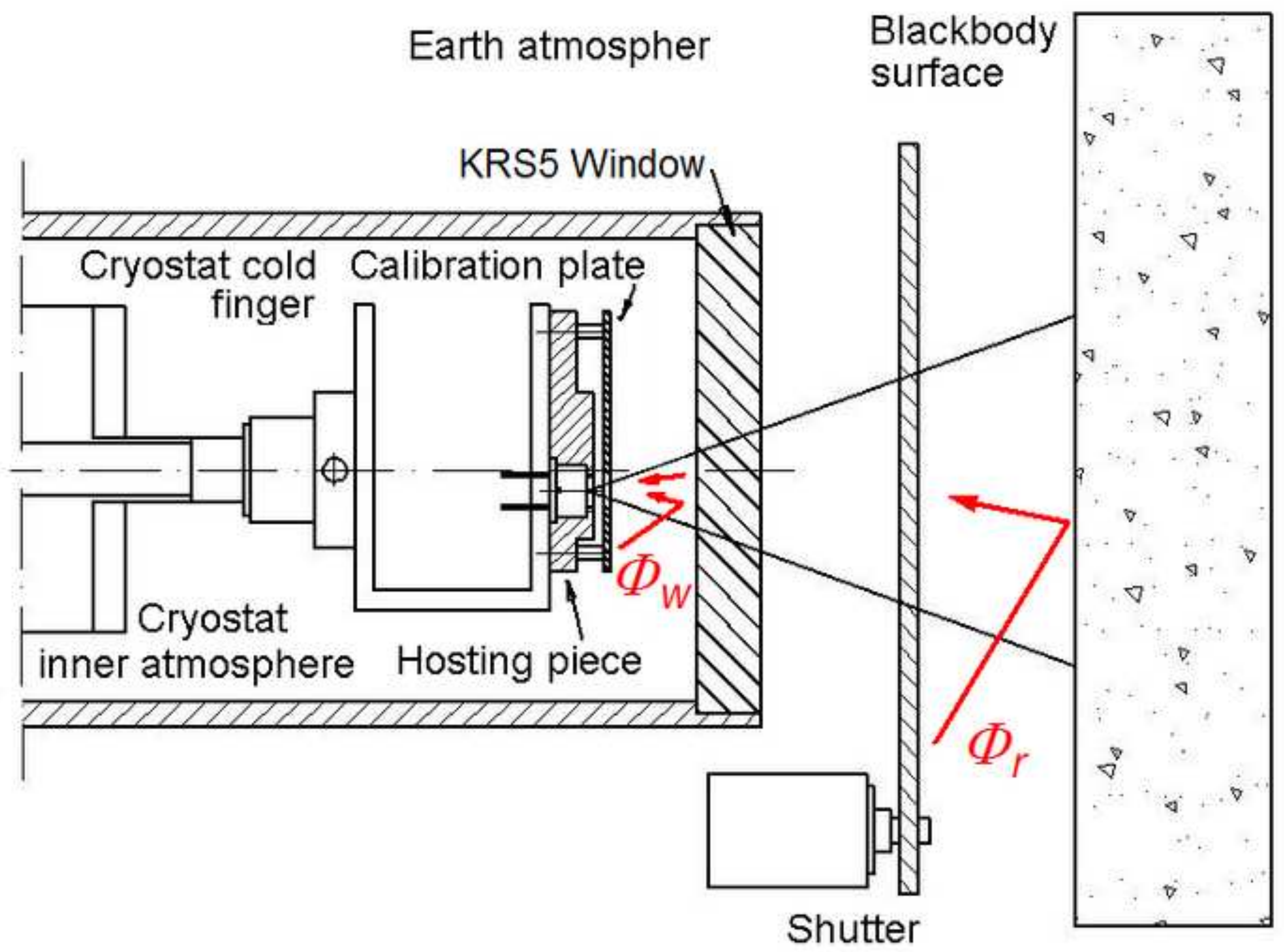




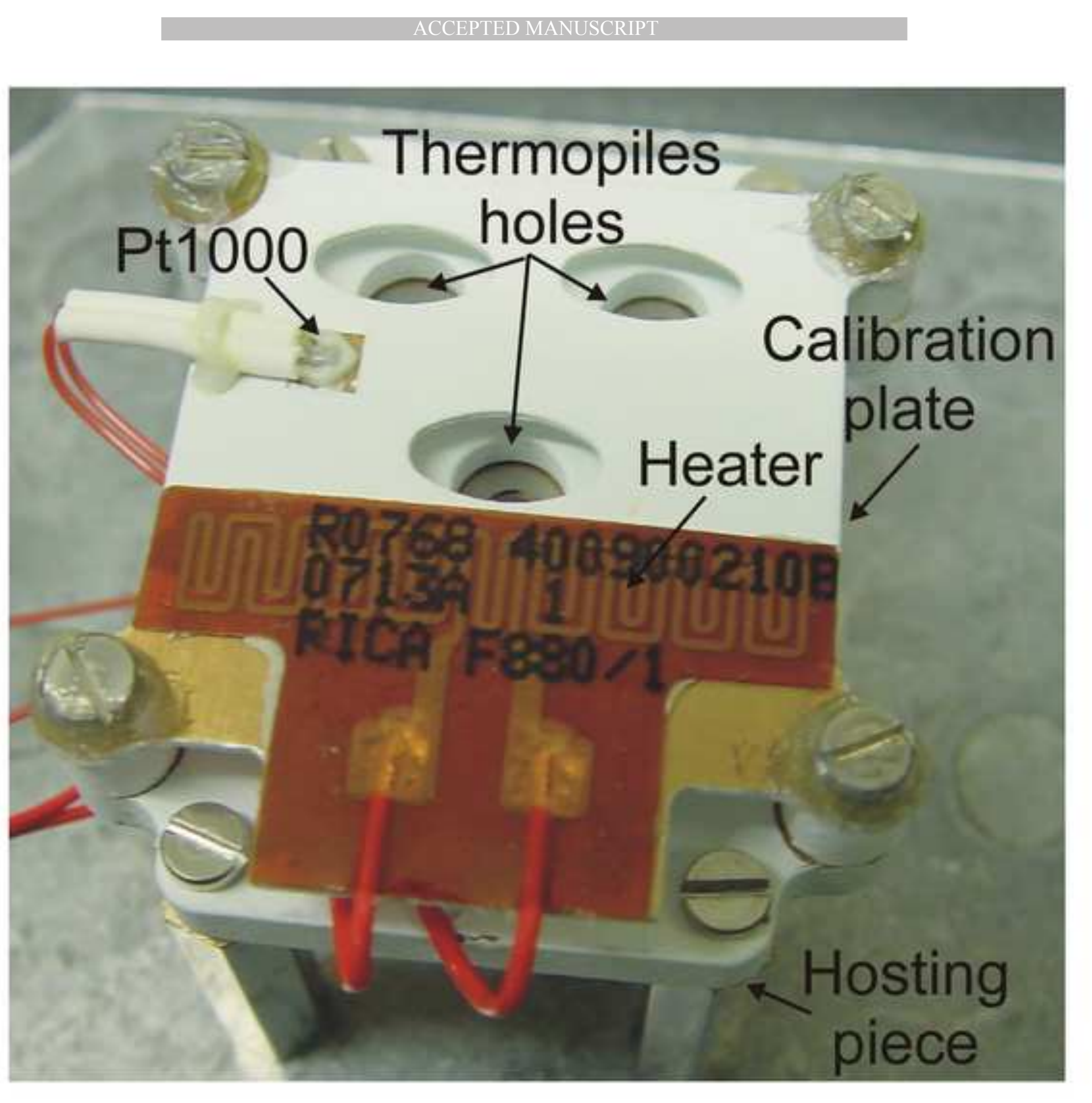

Thermopiles

Heater

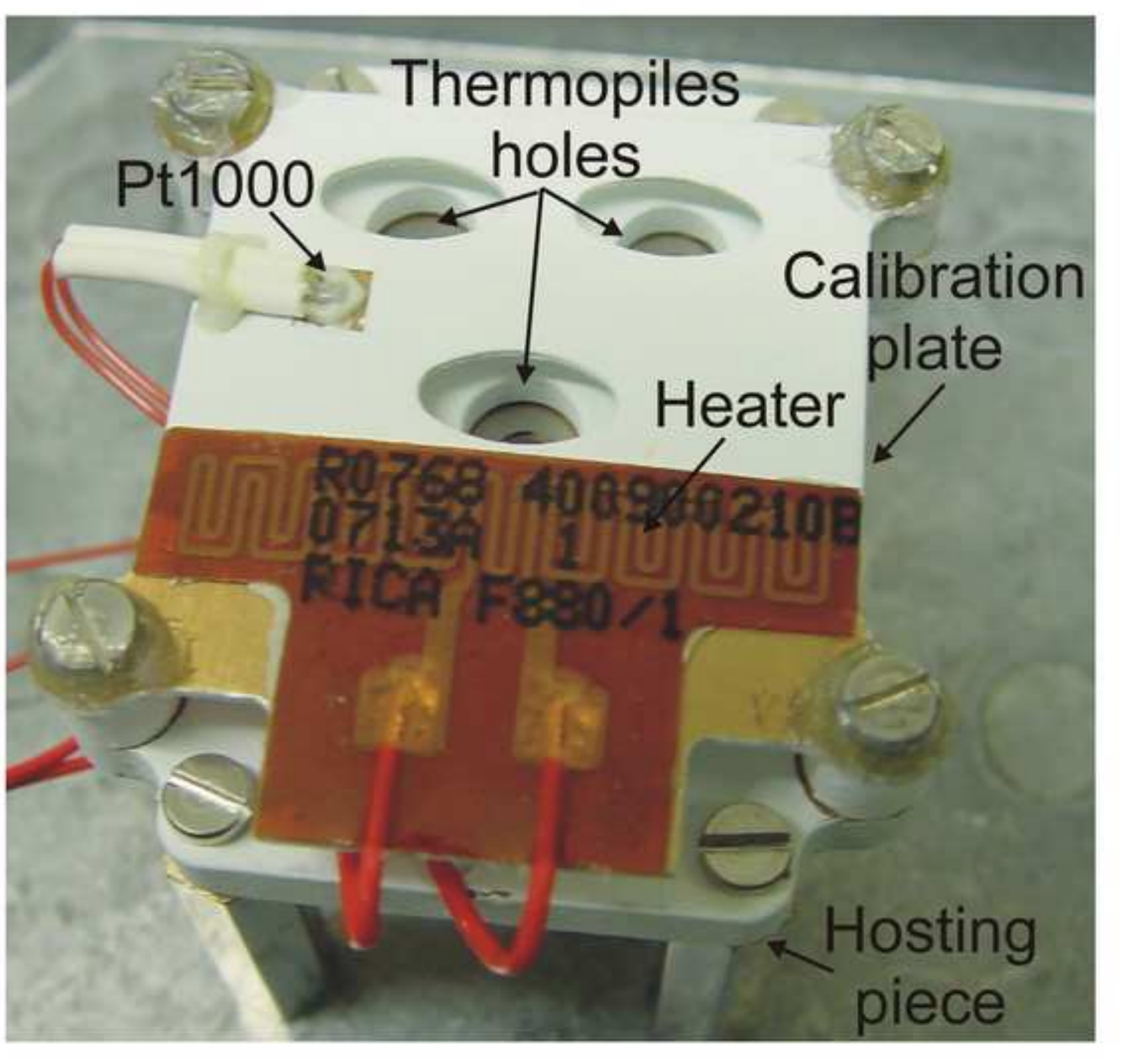
. 


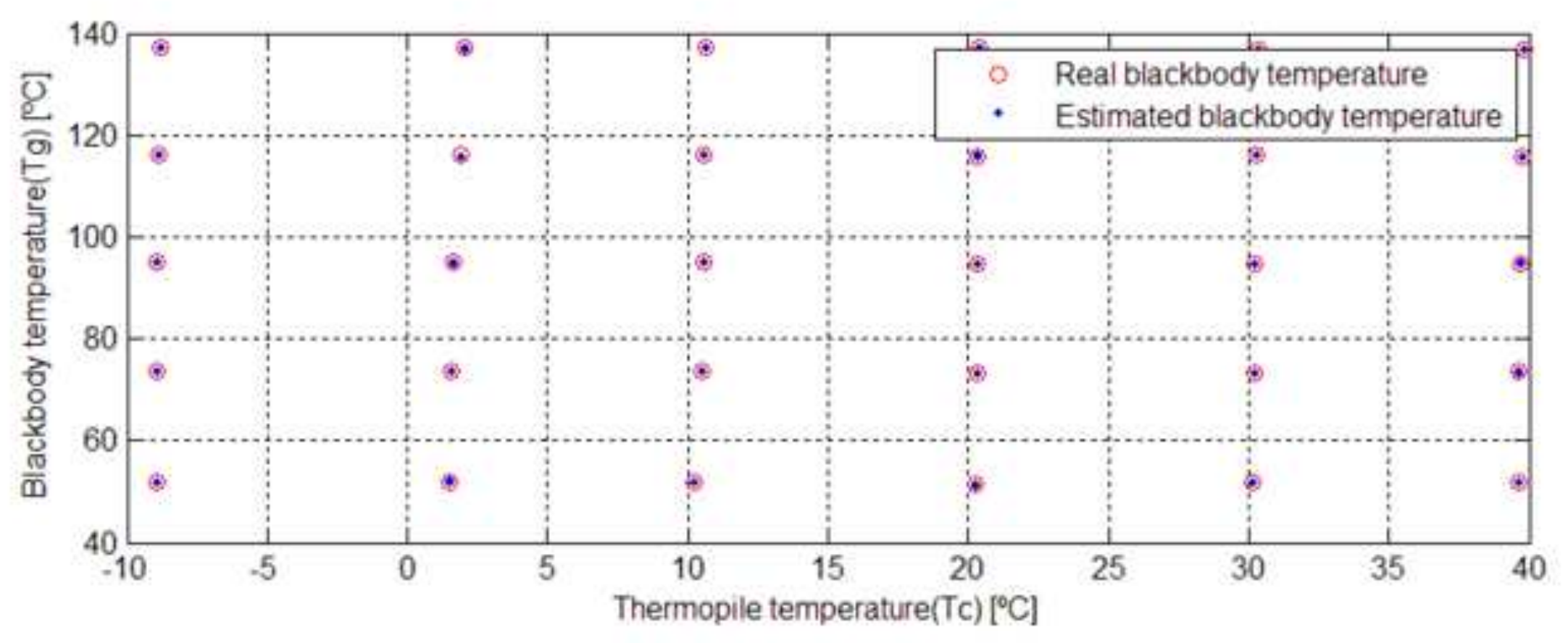

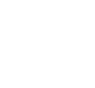




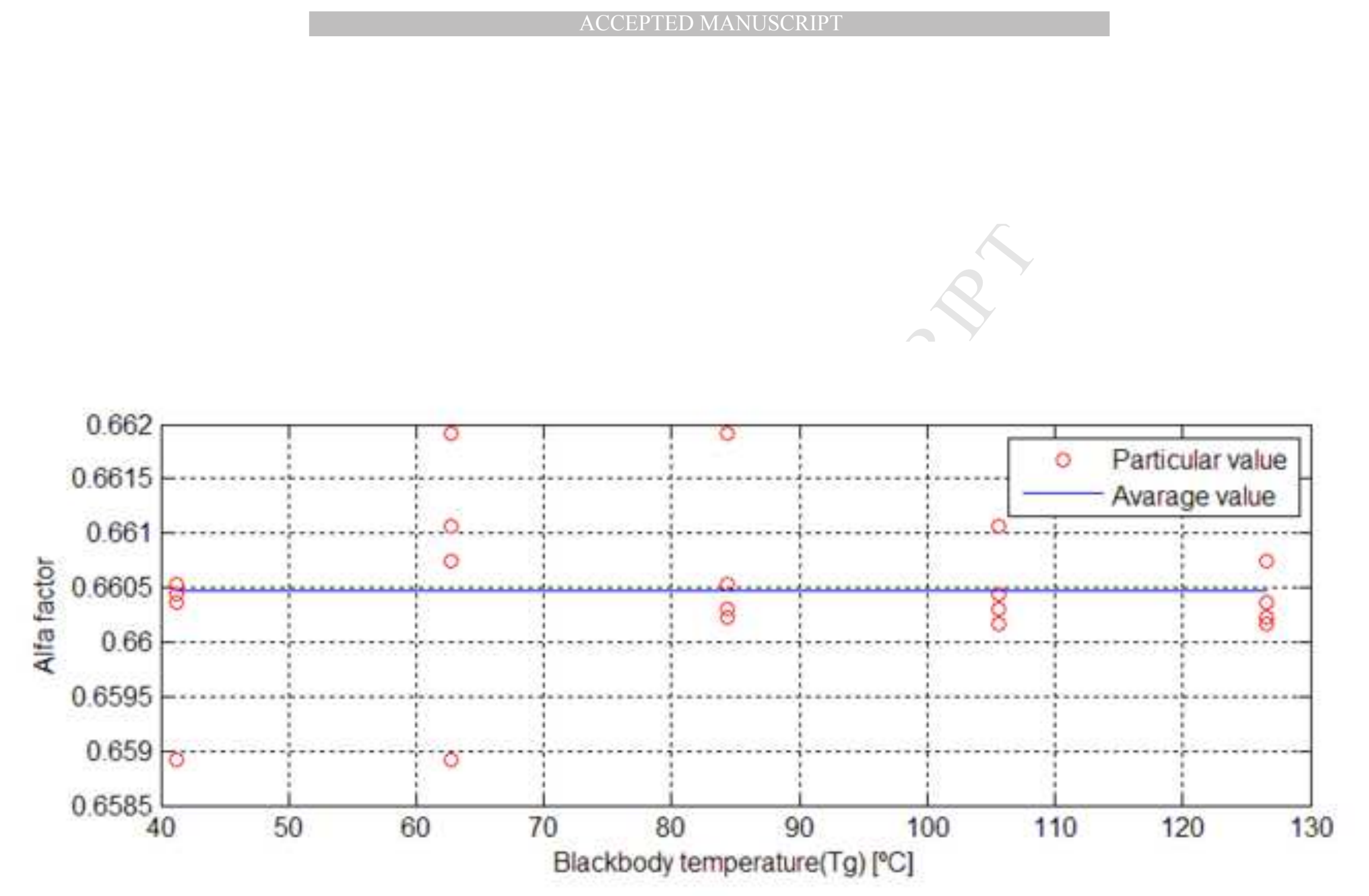

0




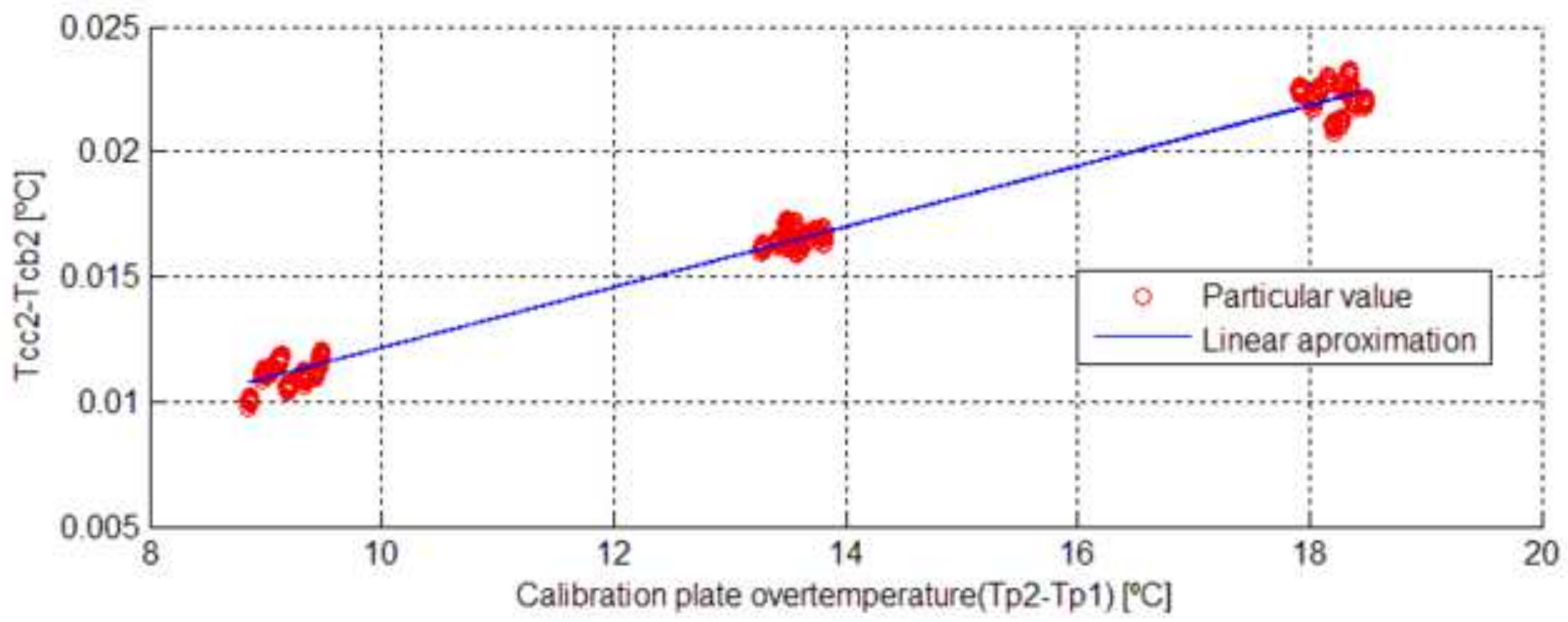




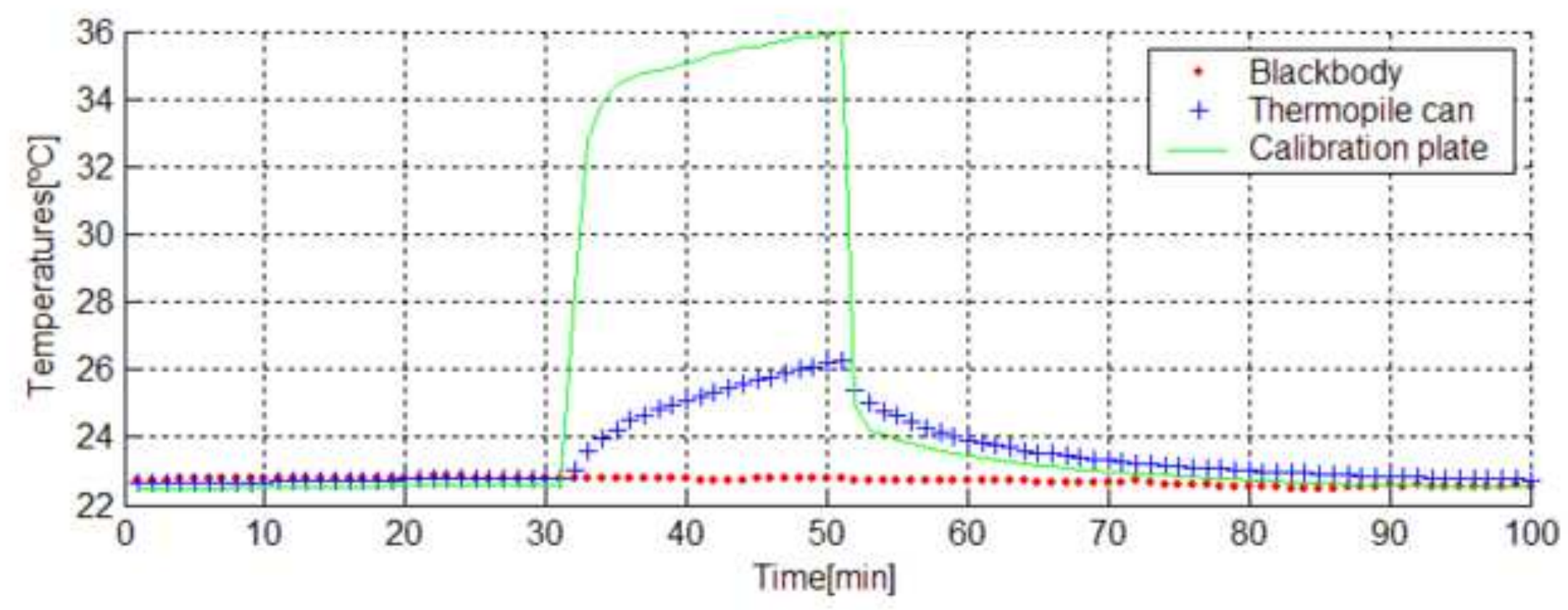




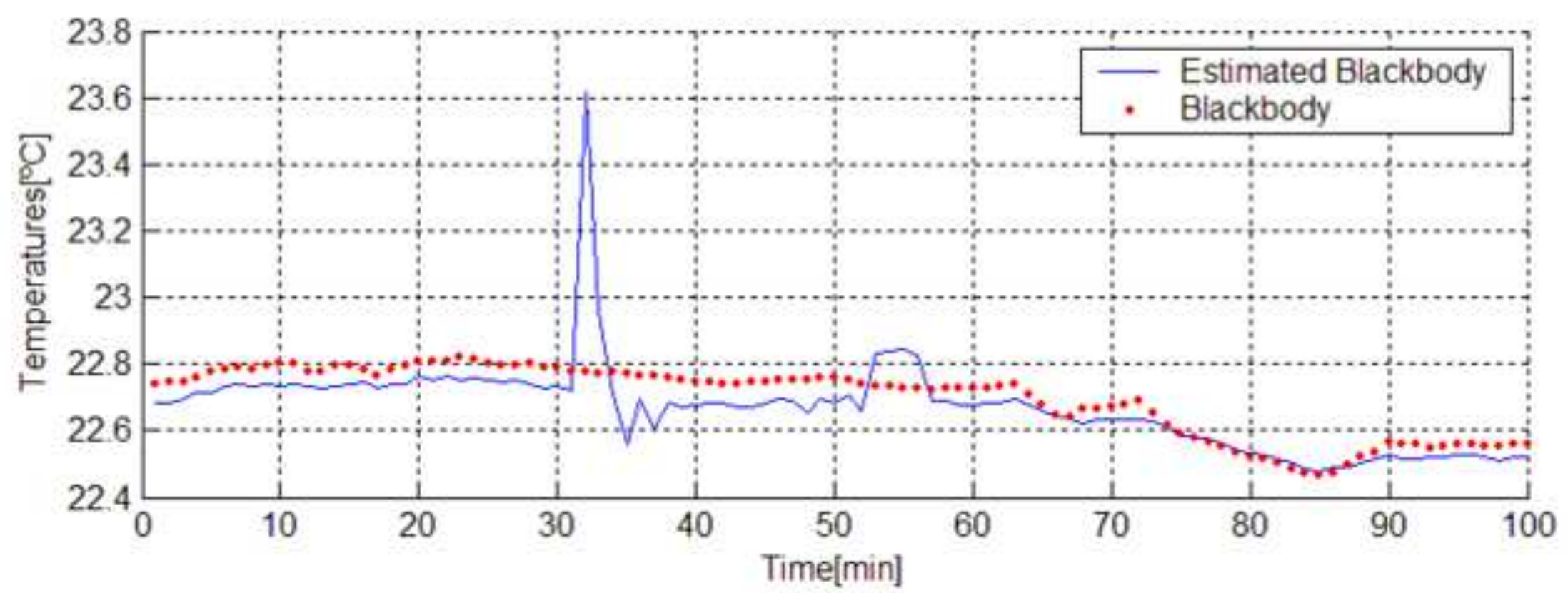

